\title{
Dendrimer-based magnetic resonance imaging agents for brain cancer
}

\author{
Ling Ding ${ }^{1,2}$, Zhenbin Lyu ${ }^{1}$, Dinesh Dhumal ${ }^{1}$, Chai-Lin Kao ${ }^{3,4}$, Monique Bernard $^{2}$ and Ling Peng ${ }^{1 *}$
}

\begin{abstract}
Brain cancer is one of the most lethal and difficult-to-treat cancers because of its physical location and biological barriers. The mainstay of brain cancer treatment is surgical resection, which demands precise imaging for tumor localization and delineation. Thanks to advances in bioimaging, brain cancer can be detected earlier and resected more reliably. Magnetic resonance imaging (MRI) is the most common and preferred method to delineate brain cancer, and a contrast agent is often required to enhance imaging contrast. Dendrimers, a special family of synthetic macromolecules, constitute a particularly appealing platform for constructing MRI contrast agents by virtue of their well-defined three-dimensional structure, tunable nanosize and abundant surface terminals, which allow the accommodation of high payloads and numerous functionalities. Tuning the dendrimer size, branching and surface composition in conjunction with conjugation of MRI functionalities and targeting moieties can alter the relaxivity for MRI, overcome the blood-brain barrier and enhance tumor-specific targeting, hence improving the imaging quality and safety profile for precise and accurate imaging of brain tumors. This short review highlights the recent progress, opportunities and challenges in developing dendrimer-based MRI contrast agents for brain tumor imaging.
\end{abstract}

Keywords: dendrimer, contrast agent, blood-brain barrier, tumor targeting, magnetic resonance imaging

\section{BRAIN CANCER AND THE BLOOD-BRAIN BARRIER}

Brain cancer is one of the most lethal forms of cancer.
Although it represents only $2 \%-3 \%$ of all tumors diagnosed $[1,2]$, the incidence of brain tumors has risen over the past decades. In general, brain tumors encompass primary brain tumors and secondary brain tumors: tumors that start in the brain are referred to as primary brain tumors, whereas tumors that stem from other part of the body and then spread to the brain are called metastatic or secondary brain tumors. Brain cancer is heterogeneous and can be classified according to the cell origin and the tumor grade $[3,4]$. Among the different brain tumors, glioblastoma is the most common in adults, accounting for $35 \%-40 \%$ of malignant brain cancer. Glioblastoma is very aggressive and has a poor prognosis. Although incremental improvements in survival have been achieved during the past decades, there is no effective cure for glioblastoma. The median length of patient survival is only $15-18$ months, and just $15 \%$ of patients survive beyond five years [5].

Surgical resection is the main method of brain cancer treatment, followed by concurrent radiotherapy and chemotherapy [3]. Complete tumor resection is often difficult because of the heterogeneous and infiltrative nature of brain tumors. In addition, tumor lesions are usually hard to distinguish from the healthy brain tissue, and they are frequently located in the areas that control movement, speech, memory, cognition, etc. Therefore, extra tissue cannot be removed during brain tumor resection, unlike other solid tumors, because of the detrimental impact it would have on the neurological and cognitive functioning of the patient [6]. It is therefore of paramount importance to precisely localize and delineate

\footnotetext{
${ }^{1}$ Aix-Marseille Université, CNRS, Centre Interdisciplinaire de Nanoscience de Marseille, «Equipe Labellisée Ligue Contre le Cancer», Marseille, France

${ }^{2}$ Aix-Marseillle Université, CNRS, Centre de Résonance Magnétique Biologique et Médicale, Marseille, France

${ }^{3}$ Department of Medicinal and Applied Chemistry, Kaohsiung Medical University, Kaohsiung, Taiwan, China

${ }^{4}$ Department of Medical Research, Kaohsiung Medical University Hospital, Kaohsiung, Taiwan, China

* Corresponding author (email: ling.peng@univ-amu.fr)
} 


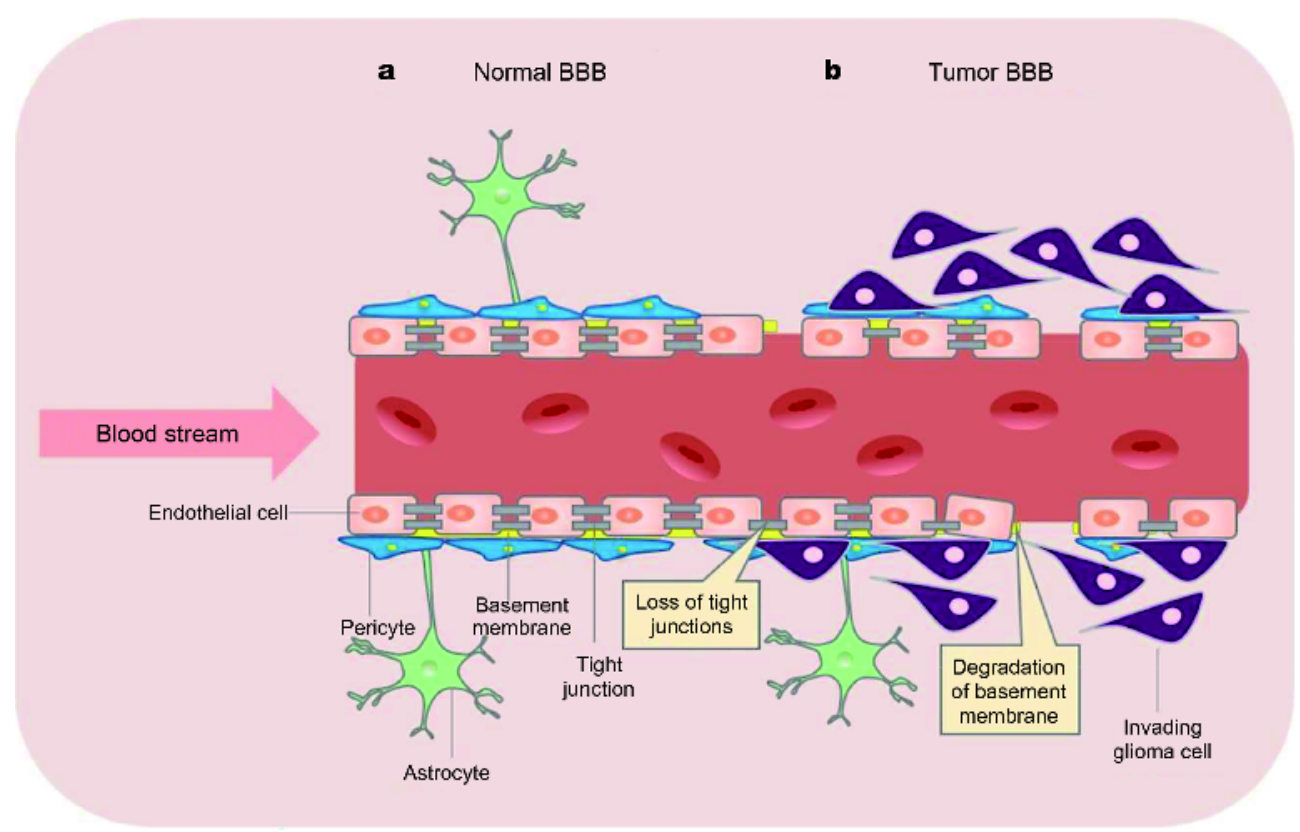

Figure 1 Cartoon presentation of the BBB (a) in normal brain and (b) in brain cancer. In the healthy brain, vascular endothelial cells form the BBB through tight junctions, pericytes and astrocytic endfeet that surround the blood vessels. In brain tumors, glioma cells migrate along the blood vessels, thus displacing the astrocytic endfeet, disrupting the tight junctions on the endothelial cells, and degrading the basement membrane surrounding the blood vessels.

brain tumors in order to allow the maximal safe resection, in which as much tumor tissue is removed as possible without causing harm to the patient [7].

However, the delivery of imaging and therapeutic agents is the greatest challenge for the diagnosis and treatment of brain cancer because of the blood-brain barrier (BBB) (Fig. 1). The BBB is a natural defense system composed of brain capillary endothelial cells alongside astrocytes and pericytes (Fig. 1a), which together regulate the passage of nutrients, electrolyte ions, metabolites, etc., while blocking unwanted harmful and foreign substances from entering into the brain, in order to keep the brain working under normal conditions [8-10]. Although the BBB is crucial for protecting the brain and maintaining its normal function, it is a nightmare for the management of brain diseases since it greatly limits the transport of therapeutic and imaging agents-both low and high molecular weight-into the brain. This is because the healthy BBB has continuous tight junctions, a lack of fenestrations and low levels of pinocytotic uptake as well as high efflux activity, etc. (Fig. 1a). It is worth noting that the human brain has high vascular density, and hence brain cells can easily be reached by circulating drugs, as long as these drugs can effectively cross the BBB.

Fortunately for brain cancer, the $\mathrm{BBB}$ is disrupted as tumor growth progresses (Fig. 1b) $[11,12]$. This is because growing tumors gradually invade and infiltrate the surrounding brain parenchyma, causing a progressive deterioration of the $\mathrm{BBB}$, which enhances the permeability. Also, the abnormal angiogenesis and the uncontrollable cancer cell proliferation cause the tumor blood vessels to become tortuous, poorly organized and leaky [13-15], further damaging the $\mathrm{BBB}$ and hence enhancing the permeability. This compromised BBB can be exploited for effective delivery of therapeutic and imaging agents to the brain, which is a challenging but important task for brain cancer management [10].

It is worth mentioning that several endogenous transport mechanisms, which regulate the passage of various substances across the $\mathrm{BBB}$, can be exploited to deliver therapeutic and imaging agents across the BBB. These transport mechanisms can be classified as passive diffusion, carrier-mediated transcytosis, adsorptive-mediated transcytosis and receptor-mediated transcytosis (Fig. 2). Specifically, water, oxygen and lipophilic small molecules are able to pass through the BBB via passive diffusion (Fig. 2a), whereas glucose, amino acids and some ions are transported across the BBB by the corresponding "carrier" proteins, a process which is referred to as carriermediated transcytosis (Fig. 2b). Some cationic molecules can be adsorbed onto negatively charged components at the luminal surface of endothelial cells and are then 


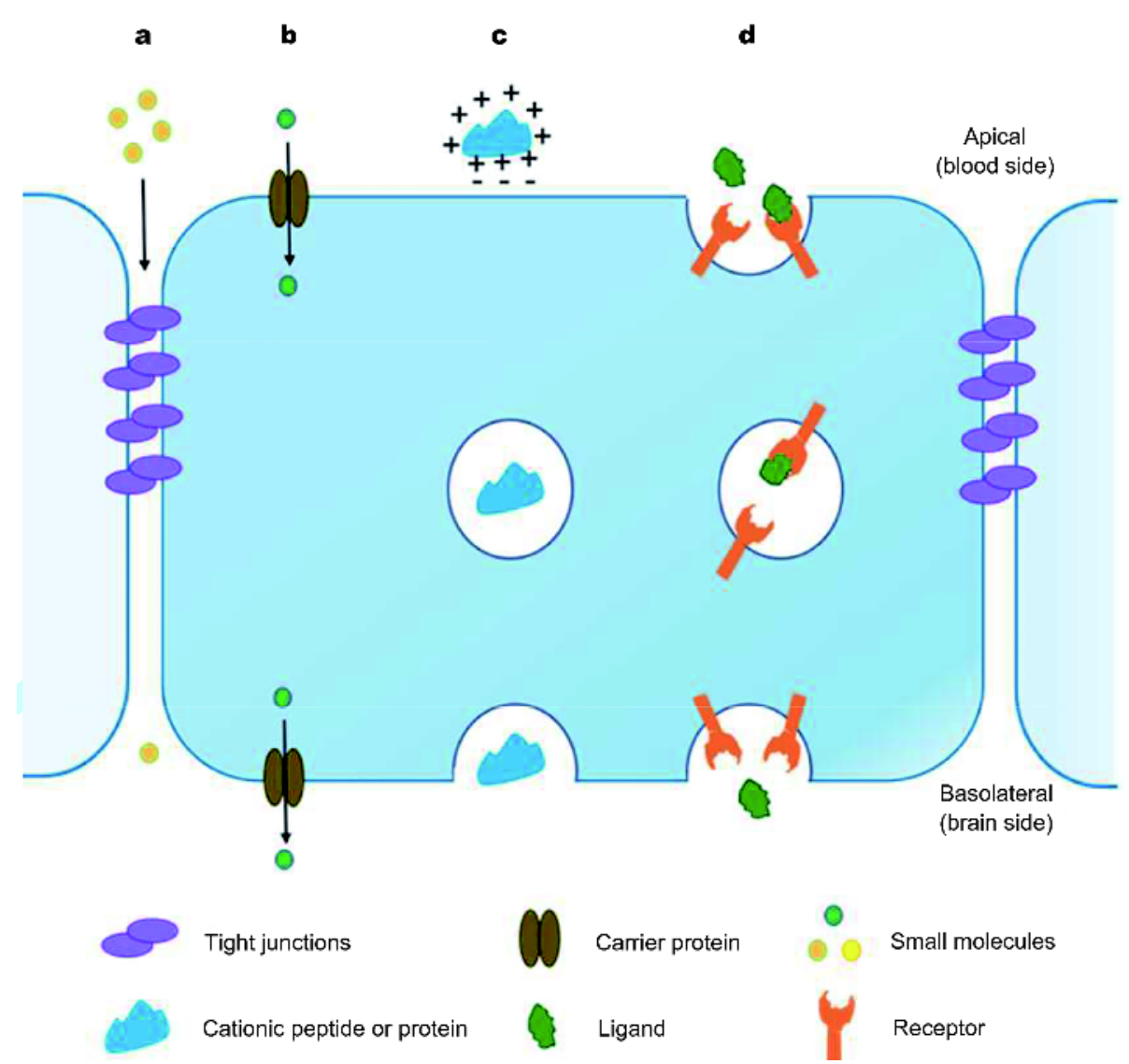

Figure 2 Endogenous transport mechanisms which regulate the passage of various substances across the BBB: (a) passive diffusion; (b) carriermediated transcytosis; (c) adsorptive-mediated transcytosis; and (d) receptor-mediated transcytosis.

exocytosed at the abluminal surface, a process called adsorptive-mediated transcytosis (Fig. 2c). Also, certain molecules are able to interact with receptors located on the surface of the BBB endothelium and are escorted to the brain cells via receptor-mediated transcytosis (Fig. $2 \mathrm{~d})$. Indeed, receptor-mediated transcytosis, adsorptivemediated transcytosis and carrier-mediated transcytosis have been frequently used to transport imaging and therapeutic agents across the BBB for the treatment of brain cancer.

\section{MAGNETIC RESONANCE IMAGING AND CONTRAST AGENTS}

Magnetic resonance imaging (MRI) is, among various methods to image brain tumors, the most common and preferred technique to localize the tumor site and determine the tumor size [16-18]. This is because MRI provides exquisite anatomical images and functional information with high spatial resolution in a noninvasive manner yet without harmful radiation $[19,20]$. MRI is therefore very important for diagnosis and preoperative imaging of brain cancer. Recently, MRI has also been applied during surgery for real-time localization of brain tumors in situ in order to perform the maximal safe tumor resection [7]. Consequently, precise and reliable MRI is crucial for successful diagnosis, tracking and resection of brain tumors.

Medical MRI is based on the principles of nuclear magnetic resonance (NMR) where the spatial distribution of magnetically active nuclei in the tissue is used to generate an MRI image. Similar to NMR, MRI can utilize NMR-active nuclei, including ${ }^{1} \mathrm{H},{ }^{11} \mathrm{~B},{ }^{13} \mathrm{C},{ }^{19} \mathrm{~F}$, and ${ }^{31} \mathrm{P}$, as a source of signal. Among them, water hydrogen nuclei $\left({ }^{1} \mathrm{H}\right)$ are the most commonly used in current clinical MRI. The basis of the ${ }^{1} \mathrm{H}$-MRI signal is the precession of water hydrogen nuclei after they are excited by radiofrequency pulses in a static magnetic field with superimposed magnetic field gradients providing spatial localization. The generated signal contains information on the density, environment and spatial localization of water hydrogen 
a<smiles>O=C(O)CN(CCN(CC(=O)O)CC(=O)O)CC(=O)O</smiles>

DTPA<smiles>O=C(O)CN1CCN(CC(=O)O)CCN(CC(=O)O)CN(CC(=O)O)CC1</smiles>

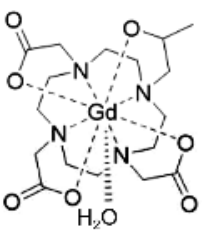

Gd-HP-DO3A

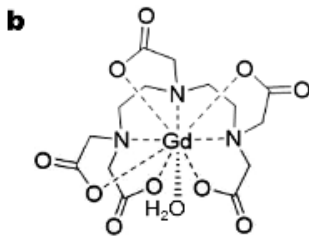

Gd-DTPA

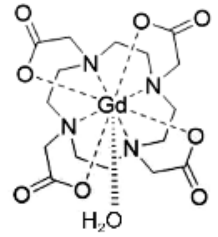

Gd-DOTA

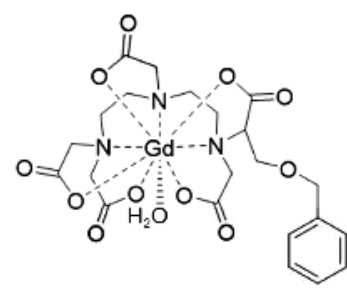

Gd-BOPTA

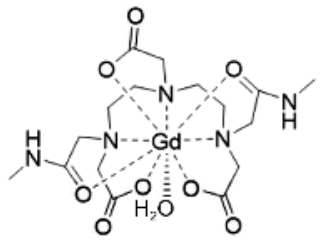

Gd-DTPA-BMA



Mn-DPDP

Figure 3 (a) Structures of the chelators DTPA and DOTA, which are commonly used to complex $\mathrm{Gd}^{3+}$ for MRI. (b) Clinically used MRI contrast agents. The $\mathrm{Gd}^{3+}$-chelates include one coordinated water molecule.

nuclei, which will be reconstructed into images. Often the endogenous magnetic resonance differences between various tissues are small, and therefore a contrast agent is used in order to enhance the imaging contrast so that the tissue of interest can be distinguished from its surroundings. Such contrast agents provide more detailed and accurate morphological and functional information, which results in improved tissue contrast, precise characterization of lesions, and better evaluation of the abnormalities [21].

MRI contrast agents can be classified as paramagnetic and superparamagnetic $[21,22]$, and the most important feature of a contrast agent is its relaxivity $(r)$, which relates to its capacity to generate imaging signal contrast. Paramagnetic agents such as gadolinium $\left(\mathrm{Gd}^{3+}\right)$ and manganese $\left(\mathrm{Mn}^{2+}\right)$ increase both the longitudinal relaxation rate $\left(R_{1}\right)$ and the transverse relaxation rate $\left(R_{2}\right)$ to a similar extent, and they exhibit "positive" (bright) contrast. These agents are named as $\mathrm{T}_{1}$ agents. Superparamagnetic agents such as iron oxide (SPIO) and ultrasmall iron oxide (USPIO) nanoparticles increase the transverse relaxation rate $\left(R_{2}\right)$ much more than the longitudinal relaxation rate $\left(R_{1}\right)$, and they display "ne- gative" (dark) contrast. These agents are referred to as $T_{2}$ agents. "Positive" contrast agents are preferable to "negative" contrast agents in the clinic because signal enhancement generated by "positive" contrast agents can be more reliably assessed than signal loss generated by "negative" contrast agents.

The majority of contrast agents currently used in the clinic are based on $\mathrm{Gd}^{3+}$ ions. This is because $\mathrm{Gd}^{3+}$ is the most paramagnetically stable metal ion, and has seven unpaired electrons with a large and symmetric magnetic moment to generate high imaging contrast. However, free $\mathrm{Gd}^{3+}$ is highly toxic, because $\mathrm{Gd}^{3+}$ is similar to $\mathrm{Ca}^{2+}$ in size, bonding and coordination chemistry [23]. By replacing or interfering with $\mathrm{Ca}^{2+}$ or other metal ions associated with biological events, $\mathrm{Gd}^{3+}$ ions can cause neurological problems and neurotoxicity, and can also accumulate in bone and liver [24,25].

In order to sequester $\mathrm{Gd}^{3+}$ and neutralize its toxicity, a variety of chelating agents have been developed such as diethyltriaminepentaacetic acid (DTPA), 1,4,7,10-tetraazacyclododecane-1,4,7,10-tetraacetic acid (DOTA) (Fig. 3a) and related derivatives. These chelators have high denticity and form complexes with $\mathrm{Gd}^{3+}$ via at least 
hepta- or octadentate coordination (Fig. 3b), hence generating thermodynamically stable and kinetically inert complexes. Also, these $\mathrm{Gd}^{3+}$-chelates will have at least one coordination site for a water molecule which is crucial for the contrast enhancement. In addition, they have a higher affinity for $\mathrm{Gd}^{3+}$ than other metal ions commonly present in the human body such as $\mathrm{Ca}^{2+}, \mathrm{Zn}^{2+}$, or $\mathrm{Cu}^{2+}$, thereby considerably reducing the toxicity of $\mathrm{Gd}^{3+}[26]$. It is worth noting that the formation of $\mathrm{Gd}^{3+}$ complexes with the macrocyclic ligand DOTA is more thermodynamically favorable than with the linear chelator DTPA. The corresponding Gd-DOTA complex thereby has superior thermodynamic stability and kinetic inertness compared to Gd-DTPA. Nevertheless, the Gd-DOTA complex has two diastereoisomeric forms that differ by the arrangement of the acetate arms and may exist in equilibrium in solution [27].

Examples of clinically used MRI contrast agents are shown in Fig. 3b [28-30]. In addition to $\mathrm{Gd}^{3+}$ agents, the $\mathrm{Mn}^{2+}$-chelate Mn-DPDP is the only other metal complex approved as an MRI contrast agent. Mn-DPDP is a weak chelate that undergoes transmetallation with endogenous metal ions $\left(\mathrm{Cu}^{2+}, \mathrm{Zn}^{2+}, \mathrm{Ca}^{2+}\right.$ and $\left.\mathrm{Mg}^{2+}\right)$ and releases free $\mathrm{Mn}^{2+}$ which is then taken up by hepatocytes, resulting in a significant contrast enhancement. $\mathrm{Mn}^{2+}$ is therefore considered a more "natural" biological cation than $\mathrm{Gd}^{3+}$. The main disadvantage of $\mathrm{Mn}^{2+}$ complexes is that they are less thermodynamically stable and kinetically inert than the $\mathrm{Gd}^{3+}$ complexes, as $\mathrm{Mn}^{2+}$ has only 6-7 coordination bonds, whereas $\mathrm{Gd}^{3+}$ often has 7-9 coordination bonds. It is also worth mentioning that the first MRI was obtained using an $\mathrm{Mn}^{2+}$ agent 30 years ago [31]. Recent interest in $\mathrm{Mn}^{2+}$ for cell tracking and as an MRI marker of ion channels has stimulated renewed efforts to develop $\mathrm{Mn}^{2+}$ chelates as molecular imaging agents.

Standard MRI is based on the excitation of hydrogen nuclei in water molecules, which vary in abundance in the different organs and tissues of the human body, thereby achieving imaging contrast. Recently, chemical exchange saturation transfer (CEST) has been proposed as a novel technique for MRI [32]. To perform CEST, contrast agents bearing a functional moiety with a labile proton (such as a hydroxyl or amide group) can be used. A saturation pulse is then applied at its resonant frequency in order to attain a saturation state. The exchange of the proton on the CEST agent with a proton on a water molecule effectively transmits the saturation to water, thus decreasing the magnetic resonance signal from water and providing an indirect assessment of the concentration of the compound of interest. CEST has the ad- vantages of enabling the imaging of certain compounds at concentrations that are too low to affect the contrast of standard MRI or too low to be directly discerned at typical water imaging resolution [33]. In general, CEST agents can be classified as diamagnetic and paramagnetic. The chemical shift of labile protons in diamagnetic CEST agents is very close to that of bulk water, whereas the offset values attainable for paramagnetic (PARACEST) agents can be up to two orders of magnitude larger [32]. Consequently, PARACEST agents hold great promise for improving the specificity of MRI diagnoses.

In addition to ${ }^{1} \mathrm{H}$-MRI, ${ }^{19} \mathrm{~F}$-MRI offers the unique advantage of highly sensitive imaging because ${ }^{19} \mathrm{~F}$ has a nuclear spin of $1 / 2$ and a high gyromagnetic ratio with $100 \%$ natural abundance, Moreover, ${ }^{19}$ F-NMR chemical shifts, which span over $300 \mathrm{ppm}$, depend significantly on changes in the chemical environment. This permits specific identification of ${ }^{19} \mathrm{~F}$ agents by ${ }^{19} \mathrm{~F}$-MRI [34,35]. Therefore, fluorinated contrast agents will be valuable for biomedical applications of ${ }^{19} \mathrm{~F}$-MRI. It is of note that ${ }^{19} \mathrm{~F}$ is almost completely absent from biological systems. The signals from exogenous fluorinated agents can therefore be easily detected and recorded without background interference. The low abundance or absence of ${ }^{19} \mathrm{~F}$ in living systems means that ${ }^{19} \mathrm{~F}$-MRI requires the use of exogenous fluorinated contrast agents at concentrations higher than common for ${ }^{1} \mathrm{H}$-MRI probes.

\section{MRI CONTRAST AGENTS BASED ON DENDRIMER NANOTECHNOLOGY}

The contrast agents that are currently in common use, such as Gd-DTPA and Gd-DOTA, are small molecular agents which have limitations of rapid renal clearance, low contrast efficiency and non-specificity, etc. Nanotechnology-based macromolecular contrast agents with nanometric size have the advantage of avoiding rapid renal clearance, thus prolonging the circulation half-life. Also, macromolecular contrast agents have greater relaxivity than low molecular weight agents because of their increased molecular weight and steric hindrance. In addition, they can also be conjugated with a targeting moeity for specific imaging. Consequently, a plethora of various natural and synthetic organic and inorganic nanomaterials differing in size, shape, structure, chemistry and architecture have been explored for use as MRI contrast agents [36-39].

Among the different nanosystems established for MRI, dendrimers are of particular interest because of their welldefined molecular structure and multivalent cooperativity confined within a nanosize volume $[36,37,40-42]$. Den- 


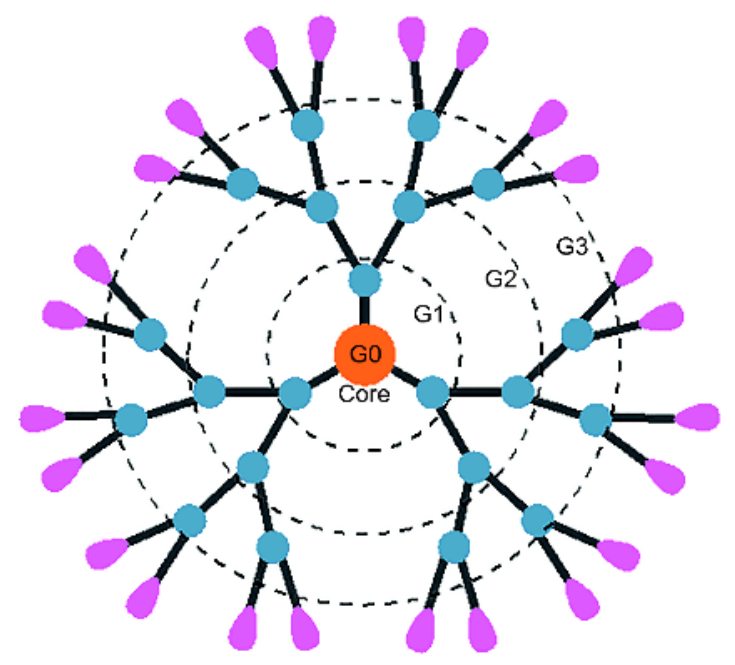

Figure 4 Cartoon illustration of a dendrimer composed of a central core, the repetitive branching units which form the generations and the terminal groups on the dendrimer surface. The central core itself is generation $0\left(G_{0}\right) ; G_{1}, G_{2}$ and $G_{3}$ refer to dendrimers of generation 1,2 and 3 , respectively.

drimers have a unique dendritic architecture with regular cascade-branched repeating units emanating from a focal point and abundant terminal groups on the surface (Fig. 4) [43-46]. A large amount of contrast agent entities can be loaded either at the dendrimer terminals or in the interior, hence increasing the imaging enhancement. Also, dendrimer contrast agents have greater relaxivity than small molecular agents, and their relaxivity can be tuned and modulated by changing the dendrimer generation. Modifications on the dendrimer surface can further affect the relaxation of water protons, by varying parameters such as hydration, hydrogen binding, and diffusion. It is also possible to adjust the proximity of water protons to the central paramagnetic ions by tuning the distance of water molecules to the dendrimer surface. All these modifications help to generate greater contrast than small-molecule contrast agents. In addition, dendrimers have sizes in the range of 3-10 $\mathrm{nm}$ and are nanoobject per se. Therefore, they can avoid rapid renal clearance and have a prolonged circulation half-life. It is noteworthy that dendrimers can be conveniently conjugated with a targeting moeity for specific imaging. Last but not least, different contrast agents and therapeutics can be easily integrated and accommodated within the same dendrimer to offer multimodal imaging and theranostics. We will give a short description of dendrimer contrast agents below.

Dendrimers are a special family of synthetic polymers and consist of three distinct structural components: 1) a central core, where dendrimer growth begins; 2) repetitive branching units, which allow for dendrimer growth in geometrically organized radial layers (named as generations); and 3) a large number of terminal functionalities exposed at the surface (Fig. 4). These structural features make dendrimers significantly different from traditional linear and hyper-branched polymers. Unlike conventional polymers which are often prepared via "one-pot" synthesis, dendrimers are constructed via stepwise synthesis, which results in precisely controlled structures. Dendrimer synthesis can be achieved using either divergent or convergent approaches (Fig. 5)

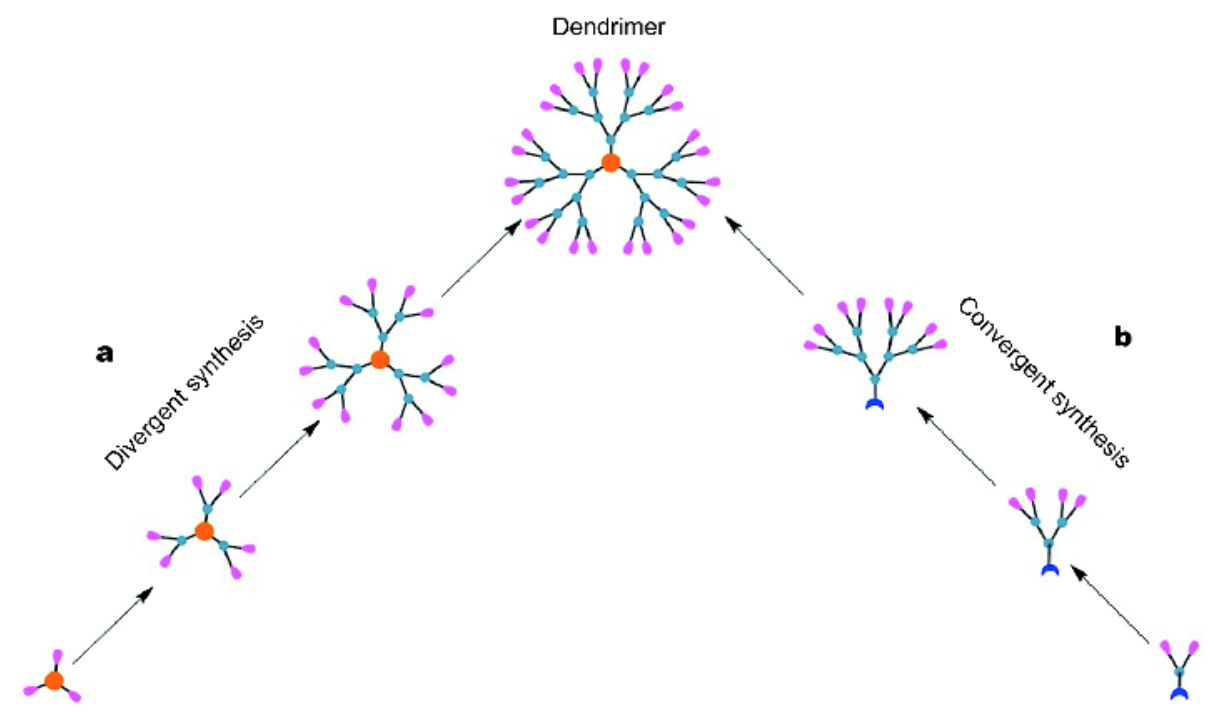

Figure 5 Cartoon presentation of dendrimer synthesis using (a) the divergent approach and (b) the convergent approaches. 
[46,47]. In the divergent strategy, the dendrimer is built from a multifunctional core and then extended outward by a series of repetitive reactions (Fig. 5a). In the convergent strategy, the dendrimer is constructed from small molecules that start at the dendrimer surface and eventually end up attached to a central core through a series of inward oriented reactions (Fig. 5b).

Most dendrimers, including the most commonly studied poly(amidoamine) (PAMAM) and poly(propylamine) (PPI) dendrimers, are prepared via divergent synthesis. Using the divergent approach, dendrimers can be synthesized to high generations of 10 or even more. Nevertheless, during the synthesis of high-generation dendrimers, structural defects are a critical concern because they are inevitably generated from incomplete reactions or various side reactions that occur due to the growing number of reactions and steric hindrance. By using the convergent approach, we can have much better control over dendrimer synthesis, and structural defects can often be avoided. However, the reactivity to reach the interior core is often considerably reduced due to the increasing steric congestion as the dendrimer generation increases. Therefore, only low-generation dendrimers can be successfully prepared using the convergent method.

A third method, called the double-stage convergent approach, has also been developed and applied for dendrimer synthesis $[47,48]$. This approach involves the synthesis of building blocks using a divergent approach followed by convergent dendrimer assembly, and hence incorporates the advantages of both divergent and convergent synthesis. In this approach, the number of reaction steps required for synthesis and purification of dendrimers can be reduced, making the preparation of higher generation dendrimers more efficient compared to traditional divergent and convergent routes.

It is worth noting that "click" chemistry has been successfully implemented in dendrimer synthesis, offering simplified and accelerated synthesis, and at the same time, diverse structural complexity [49,50]. "Click" reactions give a high yield within a short reaction time, yet can be performed under mild conditions and with high atom economy. High yield and completion of each synthetic step is crucial to maintain the purity and consistency of a dendrimer for applications ranging from molecular devices, sensors and catalysts to drugs, imaging agents and drug-delivery systems [51,52].

In 1994, Lauterbur and co-workers [53] pioneered the first study on dendrimer-based MRI agents. In that study, the DTPA chelating agent was conjugated to the terminal amine of PAMAM dendrimers of generations 2 and 6, followed by complexing with $\mathrm{GdCl}_{3}$ to deliver the dendrimer contrast agent $\mathbf{1}$ (Fig. 6). By virtue of their large molecular weight, these dendrimer contrast agents exhibited higher longitudinal relaxivities compared to the small molecular agent Gd-DTPA. Also, their blood circulation half-lives increased considerably. Importantly, these dendrimer agents showed superior in vivo imaging compared to the small molecular agent Gd-DTPA. This seminal work of Lauterbur was an important starting point for developing dendrimer-based MRI contrast agents.

Further studies using PAMAM [54,55] and PPI dendrimers (Fig. 7) [56] confirmed that the molecular and ionic relaxivities increase according to the dendrimer molecular weight and generation number, while the molar relaxivities achieve a saturation limit beyond generation 7 [55]. Nevertheless, conjugation of the $\mathrm{Gd}^{3+}$ chelate to a large dendrimer does not significantly affect the water exchange rate at the metal center, and the constants for the water exchange rate remain almost similar for all the dendrimer agents studied [57]. Also, protonated dendrimers often generate a more rigid and open structure, and hence a further increase in relaxivity [58,59]. Moreover, Ali and colleagues [60] reported a $\mathrm{pH}-$ sensitive agent based on a PAMAM dendrimer of generation 5 which bears a DOTA-derived chelating ligand, DOTA-4AmP, at the dendrimer surface. The relaxivity of this agent was more than doubled when the $\mathrm{pH}$ was changed from 9 to 6 .

Studies of the biodistribution of PAMAM dendrimer contrast agents [61-63] demonstrate that the glomerular filtration rate of smaller generation dendrimers $\left(G_{3}\right.$ to $\left.G_{5}\right)$ is high, and these smaller dendrimers are rapidly excreted, while $G_{5}$ and $G_{6}$ are retained long enough to visualize normal fine vasculature. Higher-generation dendrimers $G_{7}$ to $G_{9}$ show lower levels of renal uptake than $G_{6}$, and $G_{8}$ and $G_{9}$ exhibit much higher levels of accumulation in the liver. Consequently, $\mathrm{G}_{7}$ stands out as the most suitable agent for imaging the tumor vasculature because it is retained the longest in the circulation, allowing longer image acquisition times. Similar trends can be also found with PPI dendrimer agents [64].

The biodistribution of dendrimer agents is determined by their size, but their surface composition and modification also play a critical role in affecting the circulation half-life, pharmacokinetic and toxic profile, and targeting specificity [36]. For example, the terminal groups have a strong effect on dendrimer toxicity. Dendrimers with terminal amines are more toxic than those with terminal carboxylic acid groups [65]. Higher generations and in- 


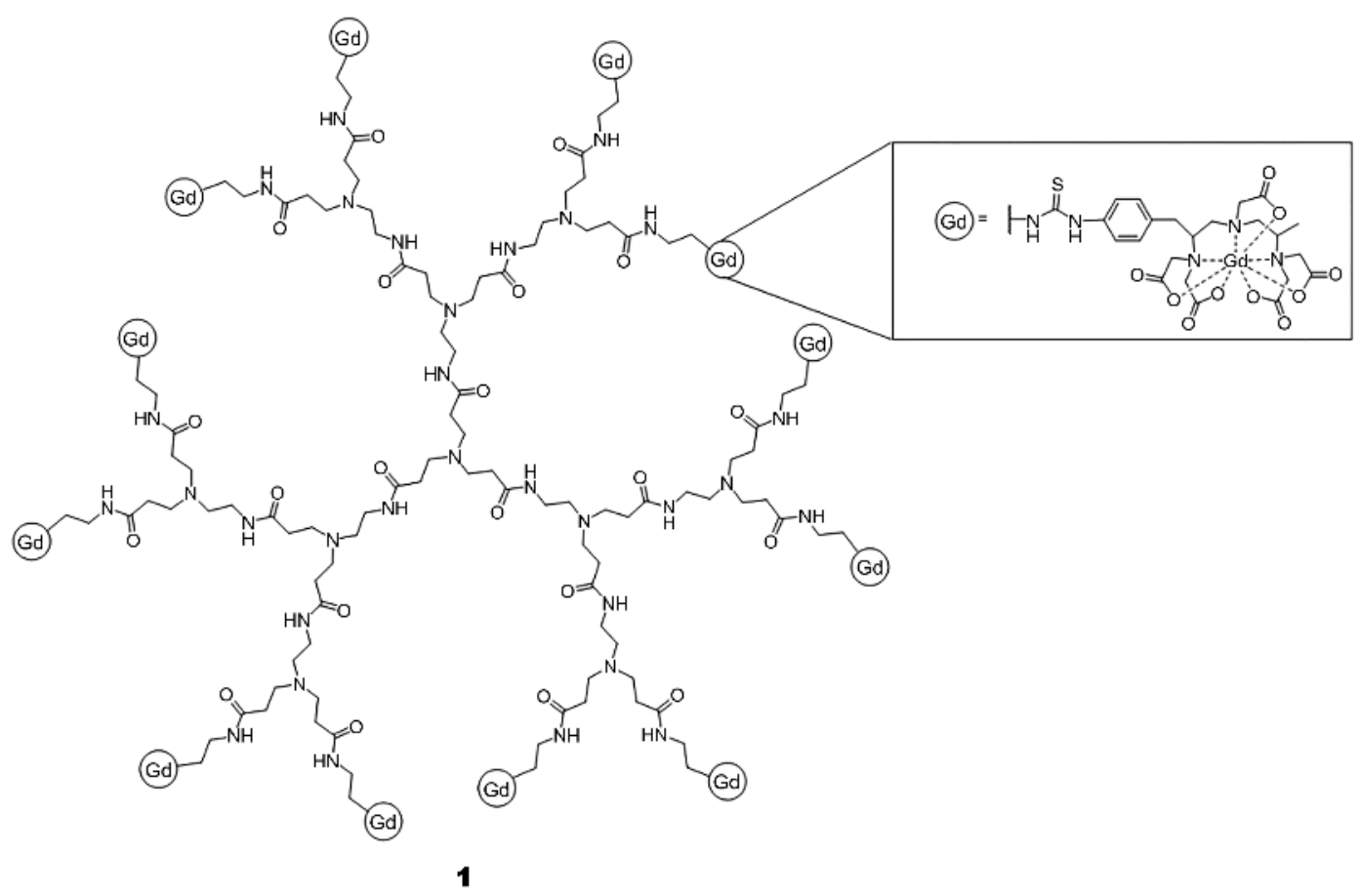

Figure 6 The first dendrimer-based contrast agent for MRI developed by Lauterbur and co-workers: Gd-DTPA functional moieties were appended to the termini of PAMAM dendrimers [53].

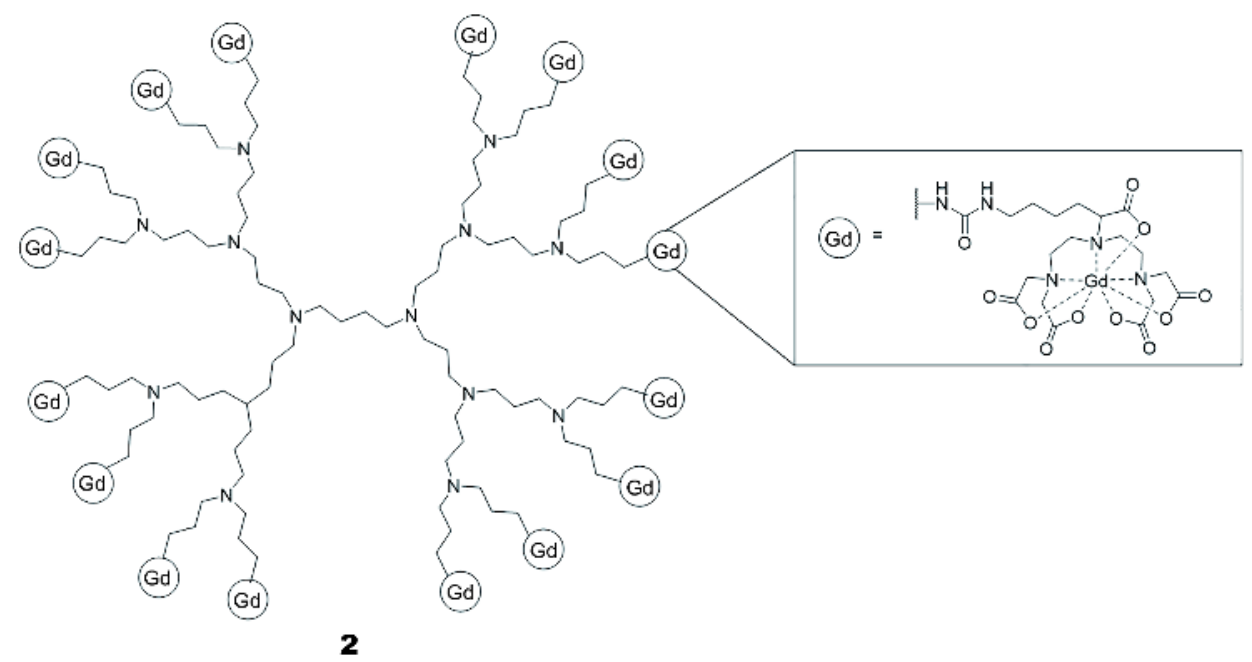

Figure 7 PPI dendrimer-based MRI contrast agent bearing Gd-DTPA terminal functionalities.

creased doses of cationic dendrimers are usually associated with more toxicity [66]. PEG is frequently appended as a surface modification to mask the surface charge of dendrimers in order to reduce toxicity, while at the same time increasing the circulation half-life, hence improving the biocompatibility. Conjugating dendrimer contrast agents with targeting moieties will modify the pharmacokinetics and biodistribution of the agents, thereby enhancing both the imaging efficiency and specificity. The development of stimulus-responsive MRI contrast agents that are sensitive to variables in the physiological and pathological microenvironment will also facilitate specific molecular imaging.

Overall, tuning the dendrimer chemistry, core, 


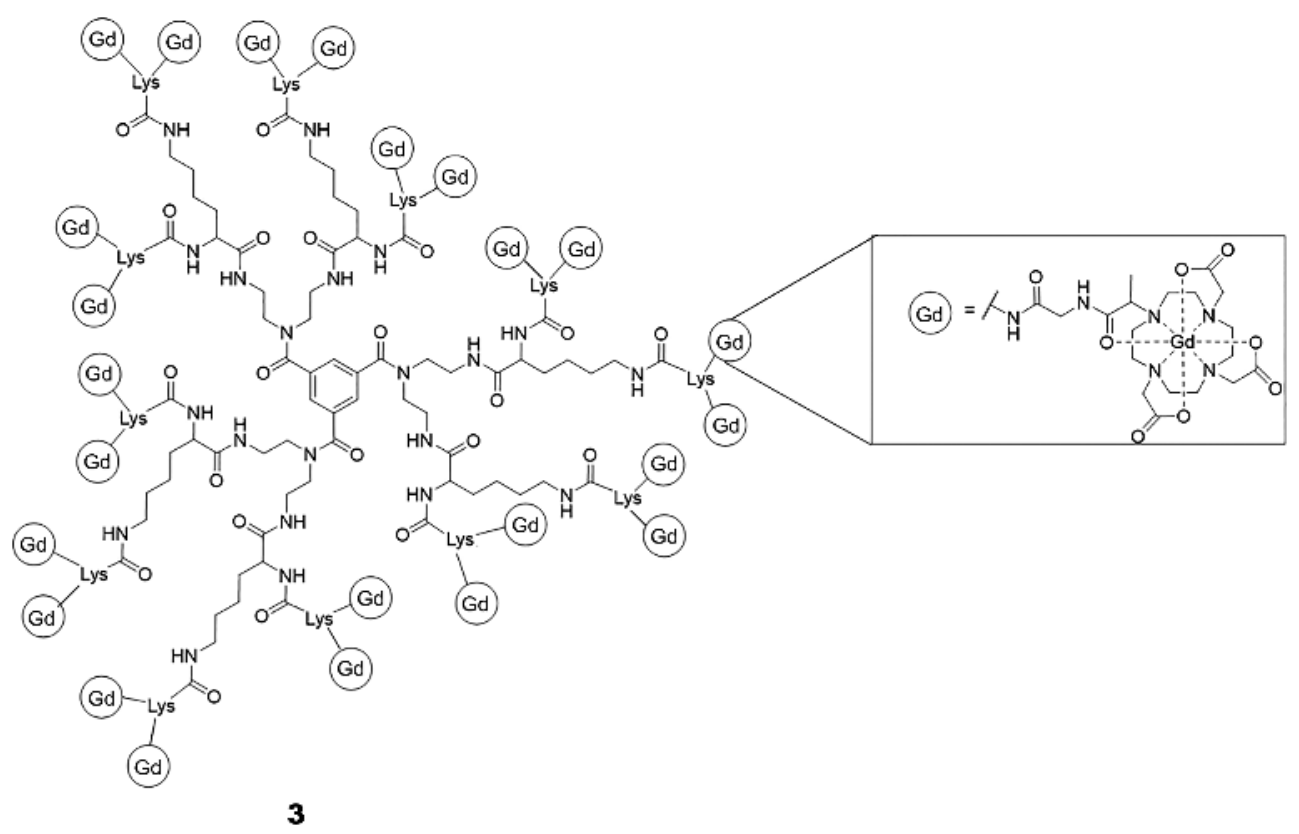

Figure 8 Gadomer-17, developed by Schering AG, has entered into clinical trials for MRI.

branching, generation and surface coating in conjunction with conjugation of contrast agents has led to different dendrimer-based MRI agents with altered relaxivities [36,37,40,41,67]. Gadomer-17 (Fig. 8), a dendrimer comprising a trimesoyl triamide core and polylysine dendritic branching with terminal payloads of Gd-DTPA [68], is the first dendrimer-based product to be clinically evaluated as a contrast agent for magnetic resonance angiography $[69,70]$. It has high vascular permeability, and is able to visualize intratumoral vasculature [71] and acute myocardial ischemia [72]. Gadomer ${ }^{\circ}-17$ showed promising results in clinical studies, but further clinical evaluation was discontinued. This contrast agent is currently commercially available for preclinical research. In this short review, we will mainly focus on dendrimerbased MRI constrast agents related to investigating and imaging brain cancer.

\section{DENDRIMER-BASED CONTRAST AGENTS FOR MRI OF BRAIN TUMORS}

An ideal dendrimer contrast agent for brain cancer imaging should possess a number of key features, namely biocompatibility, the ability to transport and protect the imaging functionality from degradation and/or rapid clearance, the potential to cross the $\mathrm{BBB}$ and reach the brain, and specific targeting and delivery of the agent to the desired cell population of interest. Also, the agent should be excreted or degraded after imaging.
As described in the introduction, brain tumors often have leaky vasculature and a disrupted BBB. This means that nanosized dendrimer agents are able to cross the $\mathrm{BBB}$ and reach tumor lesions via the leaky vasculature (Fig. 9a). Moreover, lymphatic drainage is often defective in tumor tissues. Therefore, nanosized agents which enter into the tumor tissues cannot be filtered out and will stay and accumulate there, leading to enhanced permeability and retention (EPR) in the tumor (Fig. 9a). This is also called passive tumor targeting [14,73]. EPR-mediated passive targeting results in high local concentrations of nanosized agents within tumor lesions, which in turn enhances the imaging quality, sensitivity and resolution.

Active targeting approaches that circumvent the compromised or intact $\mathrm{BBB}$ are often required for better and more efficient delivery to the brain. A promising strategy is to explore the endogenous transport mechanisms for delivery across the BBB. Due to the tight junctions, paracellular diffusion is almost impossible for macromolecular dendrimer agents. Carrier-mediated transport is also only applicable for shuttling specific hydrophilic small-molecule nutrients like amino acids and glucose. Adsorptive-mediated transport is inherently nonspecific, although it has been used to transport dendrimer agents across the $\mathrm{BBB}$. This is because adsorptive-mediated transcytosis is mainly mediated by the interaction of positively charged peptides (e.g., cell-penetrating peptides) or proteins (e.g., albumin) with negatively charged 


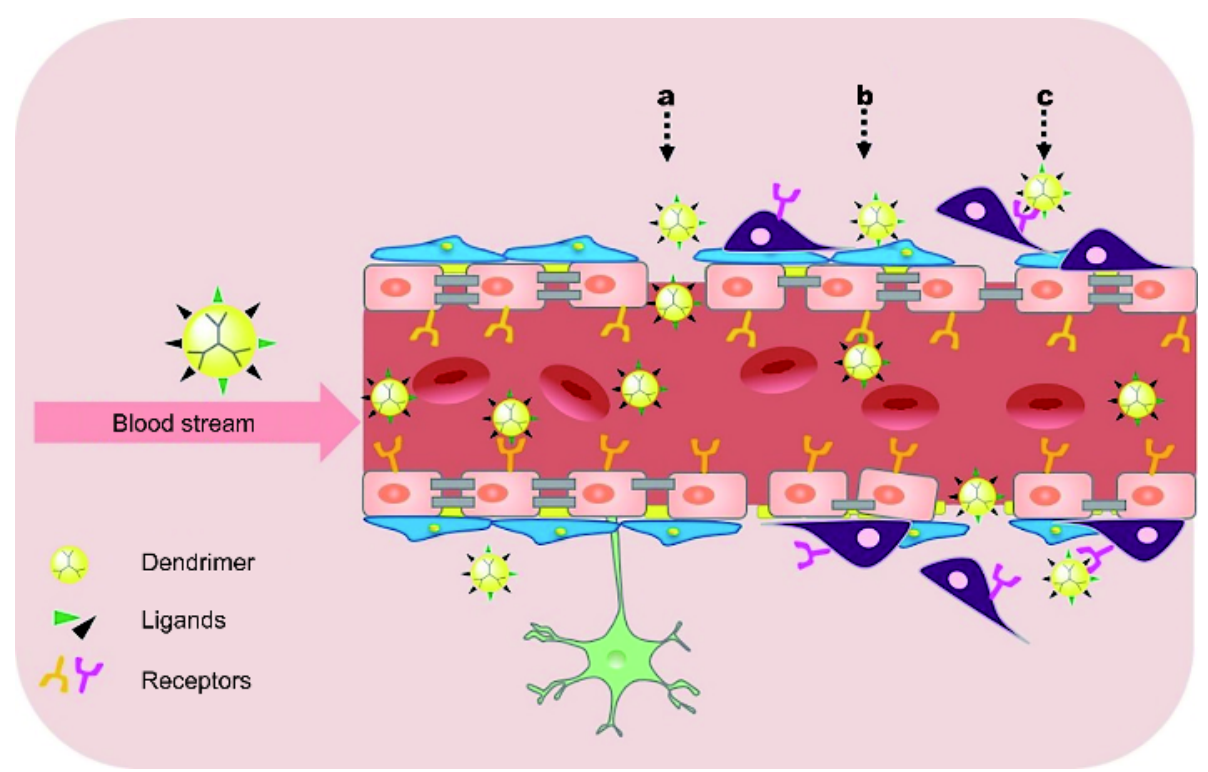

Figure 9 A dendrimer-based constrast agent can cross the BBB and reach the brain tumor via (a) the EPR effect, (b) receptor-mediated transcytosis and (c) receptor-mediated cellular uptake, triggered by ligands on the nanoparticle surface.

components on the cell membrane, triggering endocytosis and subsequent intracellular transport before eventual release into the brain in a nonspecific way [74-76]. Receptor-mediated transcytosis is the method of choice for crossing the BBB since it depends on the interaction of ligands with their specific receptors on the surface of the BBB endothelium (Fig. 9b). The discovery of several receptors that are uniquely expressed on brain capillary endothelial cells (BCECs) has made receptor-mediated transcytosis a highly promising delivery route. For example, receptor-mediated transcytosis has been demonstrated for transferrin, low-density lipoprotein receptorrelated protein (LRP), insulin, insulin-like growth factors and leptin. Taking advantage of these properties, scientists have functionalized nanoparticles with receptor targeting ligands such as transferrin [77], LRP, rabies virus glycoprotein peptide [78], and snake neurotoxin peptide [79] to up-regulate their BBB permeability.

Finally, active tumor targeting can be further realized by introducing a targeting moiety onto the delivery system, which can interact with and bind to ligands or receptors present on the cancer cell surface [80-83]. In this way, the imaging agent can be delivered specifically to the target cancer cells after crossing the BBB (Fig. 9c), thereby achieving higher delivery efficacy while sparing other cells to reduce toxicity. Different targeting agents such as antibodies, peptides and small molecular ligands have been applied to construct active targeting systems for tumor homing [84-86]. These systems can be further developed to create nanoplatforms for brain tumor targeting.

In this section, we will present examples of dendrimerbased MRI contrast agents related to brain tumor imaging. We will also report combinations of different imaging techniques for multimodal imaging and integration of therapy and imaging modalities to develop theranostics for treating brain tumors.

\section{Passive versus active targeting}

As mentioned above, nanoparticles can accumulate within tumor lesions by EPR-mediated passive targeting. In order to achieve brain tumor targeting, researchers have used specific functional entities to overcome the BBB and actively target cancer cells $[84,85,87]$. It is expected that dendrimer-based imaging agents would accumulate and be enriched at the tumor lesions via the EPR effect and/or receptor-mediated transcytosis, then bind to ligands or receptors present on the brain cancer cell surface (Fig. 9). In this way, the imaging agent can be delivered specifically to the cancer cells after crossing the $\mathrm{BBB}$, thereby achieving higher delivery efficacy while sparing healthy cells to avoid adverse effects. Below, we will highlight both passive and active targeting approaches for delivering dendrimer-based contrast agents to brain tumors with recent examples.

\section{Passive targeting}

Following the seminal work of Lauterbur on PAMAM dendrimer-based contrast reagents [53], improved con- 


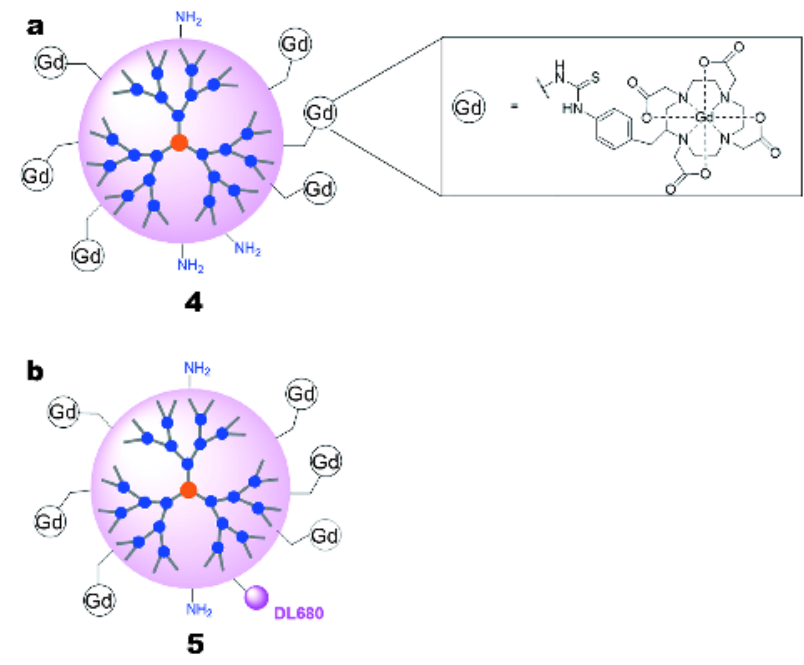

Figure 10 PAMAM dendrimers bearing (a) the contrast function GdDOTA and (b) both Gd-DOTA and the fluorescent dye DL680 at the terminals $[89,90]$.

jugation of Gd-DTPA to dendrimers was achieved using non-aqueous chemistry [88]. The $p$-NCS-benzyl functional group was used as a rigid linker to join Gd-DTPA or Gd-DOTA to the dendrimer in order to ensure that the slow rotation of the rigid dendrimer molecule can be transmitted to the surface MRI functions. Recently, a PAMAM dendrimer of generation 5 bearing the contrast function Gd-DOTA (4) at the terminals (Fig. 10a) showed glioma-specific accumulation in animal models [89,90]. This can mainly be ascribed to the defective BBB of the brain tumor and the EPR effect of the nanoparticulate agents. Furthermore, the effectiveness of MRI imaging was confirmed using in vivo and ex vivo optical imaging with the dendrimer agent (5), which carries both the GdDOTA payload and the fluorescent dye DL680 (Fig. 10b).

As we know, brain cancer disrupts the BBB as it progresses [91], and the blood-brain-tumor barrier (BBTB) is established once the BBB becomes damaged. The BBTB often contains large pores, which make the BBB more permeable to nanosized macromolecules than the endothelial barrier in the microvasculature of most normal tissues. In order to probe the pore size in the BBTB of brain tumors, Sarin et al. $[92,93]$ labelled PAMAM dendrimers of generation 5 to 8 with Gd-DTPA at the terminals, and investigated their transport in brain tumors in mouse models using dynamic contrast-enhanced magnetic resonance imaging. The size of the $G_{7}$ dendrimers bearing Gd-DTPA functions was $11 \pm 1 \mathrm{~nm}$, and that of the $G_{8}$ dendrimers was $13 \pm 1 \mathrm{~nm}$. The functionalized $\mathrm{G}_{5}-\mathrm{G}_{7}$ dendrimers with sizes less than $12 \mathrm{~nm}$ were able to pass through the pores of the BBTB in RG-2 glioma xenograft mice, whereas larger $\mathrm{G}_{8}$ dendrimers could not. In addition, dendrimers with long blood halflives were able to accumulate more effectively within glioma cells. This finding suggests that the physiological upper limit of the pore size in the BBTB of the microvasculature in malignant solid tumors is approximately $12 \mathrm{~nm}$. This is in line with the results obtained from a later study on the permeability of solid tumors using nanoparticles of different sizes [94], where the optimal size of nanoparticles for tumor penetration was found to be around $12 \mathrm{~nm}$ [95]. Therefore, effective delivery into malignant brain cancer cells can be achieved with nanoparticles less than $12 \mathrm{~nm}$ in diameter and with a long blood circulation time.

Among the various dendrimers explored, PAMAM, PPI and poly(lysine) dendrimers are most frequently studied for establishing MRI agents because of their good biocompatibility and easy chemical conjugation as well as their commercial availability [36]. Nevertheless, polyester and polyether dendrimers have also been explored for constructing MRI agents [96-98]. In addition, placing the $\mathrm{Gd}^{3+}$ ion at the dendrimer core was further proposed to increase the relaxivity (Fig. 11) [97,98]. However, the
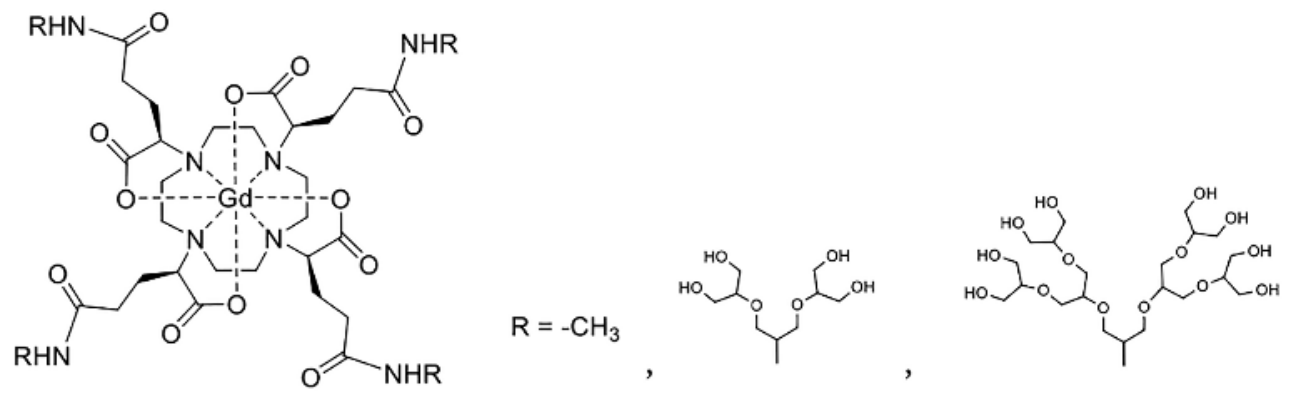

6

Figure $11 \mathrm{Gd}^{3+}$ incorporated at the dendrimer core to increase the relaxivity for MRI [97]. 


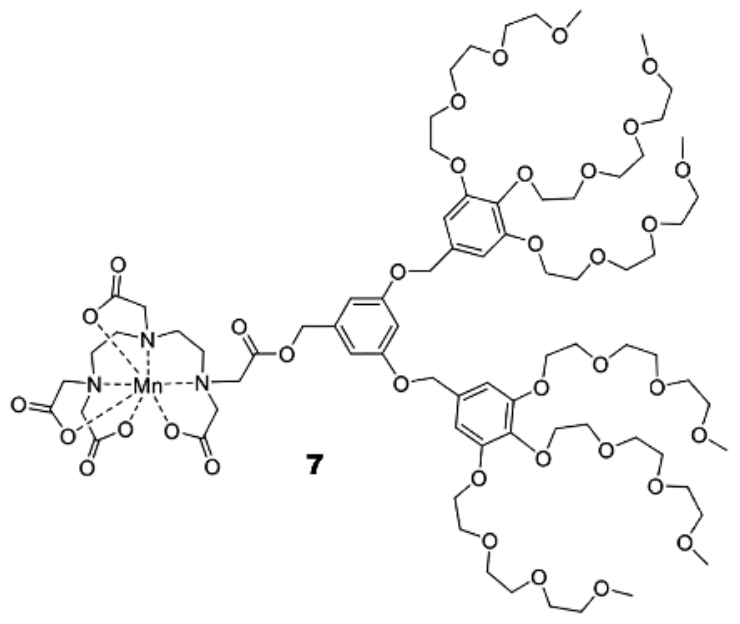

Figure 12 The dendritic manganese contrast agent 7 [99].

water exchange rate will also be slowed down, and this will tend to negate any theoretical increase in relaxivity.

Besides $\mathrm{Gd}^{3+}$, a dendritic $\mathrm{Mn}^{2+}$-enhanced contrast agent (Fig. 12) was also developed for brain targeting [99]. This is because $\mathrm{Mn}^{2+}$ has natural human biochemistry: the uptake of extracellular $\mathrm{Mn}^{2+}$ by neurons is often through NMDA receptors for glutamate and/or voltage-gated calcium channels, and the uptake of $\mathrm{Mn}^{2+}$ is directly coupled to the physiological activity and functions of the cells. $\mathrm{Mn}^{2+}$ can therefore generate contrast in a manner that depends on cellular activity, which means that $\mathrm{Mn}^{2+}$ based contrast agents have potential for investigating neuronal activation and architecture. The dendritic $\mathrm{Mn}^{2+}$ conjugate 7 displayed high water solubility and revealed neither in vitro neuronal toxicity at concentrations up to $1 \mathrm{mmol} \mathrm{L}^{-1}$ nor in vivo toxicity at $20 \mathrm{mmol} \mathrm{L}^{-1}$ in a rat model. The excellent safety profile of this dendritic manganese contrast agent is likely due to the slow release of the paramagnetic ions. Also, its relaxivity is higher than that of the commercial agent Mn-DPDP, which suggests that it may have potential applications in studying and treating brain and neurodegenerative diseases.

It is worth noting that passive targeting via the EPR effect is unlikely to be an effective and viable approach for imaging and treating brain cancer [100], because the BBB is a formidable obstacle even if it is disrupted by a developing tumor. The extravasation of nanoparticulate agents and their accumulation in the tumor via the EPR effect depends entirely on nanoparticle diffusion, which is difficult to control. Also, some agents display extremely low rates of diffusion. In addition, tumor blood vessel permeability is not homogeneous even within the same tumor. Last but not least, the elevated interstitial fluid pressure can block passive diffusion of nanoparticles into the brain parenchyma. Consequently, there is great interest in active targeting strategies for delivery of nanoparticles to brain tumors.

\section{Active targeting}

Considerable efforts have been devoted to developing active targeting approaches to overcome the $\mathrm{BBB}$ and specifically target brain cancer cells. As described above, receptor-mediated transcytosis is a naturally-occurring pathway through which endogenous and exogenous molecules can cross the BBB. Ligands that have been explored for targeting brain tumors via receptor-mediated transcytosis include transferrin, LRP, neurotoxins, peptides, nucleic acids and antibodies.

Chlorotoxin (CTX) is a 36 -amino acid peptide that specifically binds to brain glioma cells [101], thus allowing the penetration of gliomas through tissue barriers. Its targeting specificity stems from its selective binding to membrane-bound matrix metalloproteinase-2 isoforms (MMP-2) [102], which are expressed at high levels in gliomas and related cancers, but not in healthy brain cells. An ${ }^{131}$ I-labelled CTX is currently undergoing clinical trials for treatment of brain cancer [103]. Huang et al. [104] reported a dendrigraft poly-L-lysine dendrimer (DGLs) equipped with CTX as the glioma tumor targeting ligand and Gd-DTPA as the imaging payload for MRI (Fig. 13). This agent markedly enhanced the cellular uptake in C6 glioma, but not in normal cells. In addition, when mice were treated with this agent, the imaging contrast was significantly improved compared to the unmodified counterpart and the commercial control. Remarkably, the enhanced signal from this agent was maintained much longer than the controls, illustrating its potential for improving imaging and diagnosis. This discovery is particularly relevant to brain glioma, which is difficult to image and diagnose because of the BBB.

LRP mediates the transport of a variety of ligands across the BBB, such as lipoproteins, extracellular matrix proteins, proteases and protease inhibitor complexes [105]. LRP receptors are highly expressed on brain capillary endothelial cells (BCECs) and on many types of glioblastoma [106]. Angiopep-2, a 19-amino acid peptide, is a ligand of the LRP receptor and demonstrates a high BBB transcytosis efficacy [107]. For example, an angiopep-2-paclitaxel conjugate, ANG1005, has been developed to deliver the anticancer drug paclitaxel across the BBB to treat glioma. ANG1005 has been subjected to several Phase I clinical trials [108], and Phase II trials are 


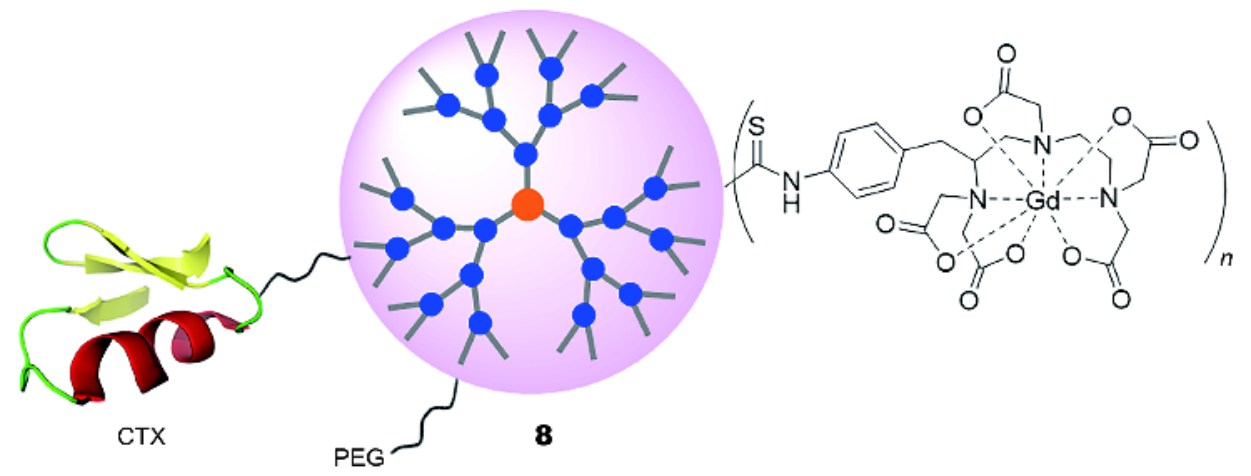

Figure 13 Dendrigraft poly-L-lysines (DGLs) conjugated with Gd-DTPA and the CTX peptide for targeted MRI of brain tumors [104].

underway (http://angiochem.com). Angiopep-2 is therefore frequently chosen as the BBB-permeable ligand for nanoparticle delivery to the brain. $\mathrm{Li}$ and co-workers [109] conjugated angiopep-2 to a dendrimer of generation 5, along with the MRI reporter Gd-DTPA and the fluorescent dyes Cy5.5 and rhodamine, to create a dual MRI/fluorescent imaging agent (Fig. 14a). This agent combines BBB permeability, passive and active targeting functions to visualize glioblastomas, and high sensitivity. It therefore holds promise for pre-operative localization of brain tumors and for making image-guided tumor resection possible during surgery.

Additionally, Li and co-workers [110] conjugated cyclic RGD peptide to the above dendrimer agents to further improve the tumor targeting and imaging quality (Fig. 14b). The RGD tripeptide is the ligand of $\alpha_{v} \beta_{3}$ integrin, which is often present on tumor cells, and is also highly expressed on activated endothelial cells of the tumor neovasculature, but not the normal vasculature [111]. Therefore, integrin is a molecular target for tumor diagnosis and therapy [112]. Dendrimer nanosystems decorated with the RGD targeting peptide showed effective delivery in glioblastoma cancer models [113,114]. The dual-targeting imaging agent $\mathbf{1 0}$ developed by $\mathrm{Li}$ and co-workers is expected to first target $\alpha_{\mathrm{v}} \beta_{3}$ integrin on the tumor vasculature via binding of the cyclic RGD peptide [115]. Increased local concentration of $\mathbf{1 0}$ within the tumor vasculature then facilitates interaction of the BBBpermeable angiopep-2 peptide with the LRP receptors on the vascular endothelial cells, which promotes transport across the BBB via LRP receptor-mediated endocytosis. After the agent 10 penetrates the $\mathrm{BBB}$, it then targets the brain tumor because the tumor cells express high levels of both $\alpha_{\mathrm{V}} \beta_{3}$ integrin and LRP receptor. In vivo imaging studies showed that $\mathbf{1 0}$ efficiently crossed the intact BBB in normal mice, and also facilitated precise detection of
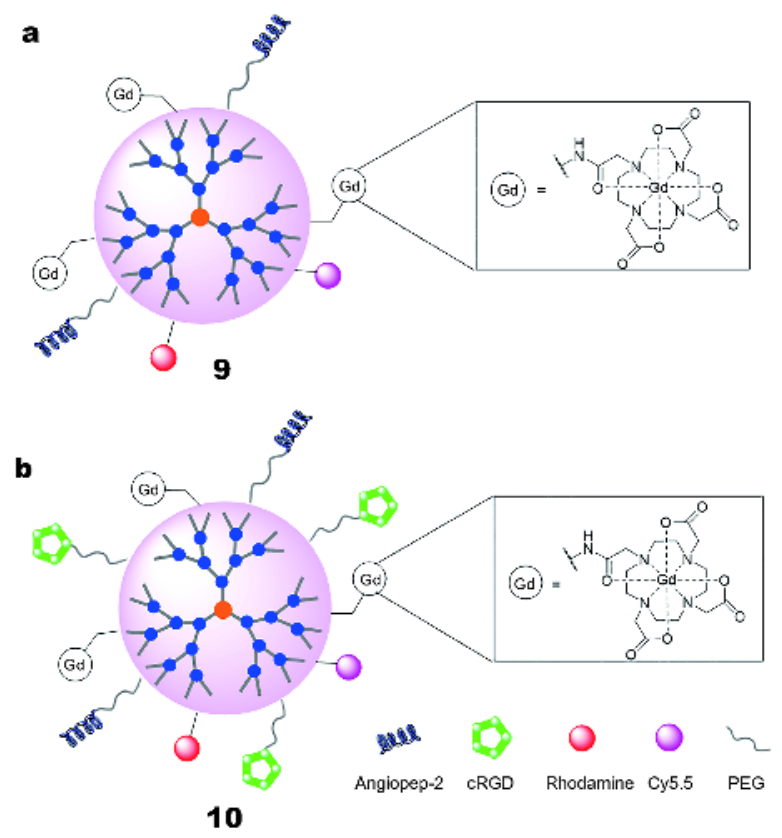

Figure 14 PAMAM dendrimers decorated with the BBB-permeable angiopep-2 peptide, the MRI reporter Gd-DTPA, and the fluorescent dyes Cy5.5 and Rhodamine, without (a) and with (b) the cyclic RGD peptide as active targeting agents to visualize brain tumors $[109,110]$.

the boundary of the orthotopic U87MG human glioblastoma xenografts with high contrast and sensitivity. Overall, this dual and sequential targeting contrast agent 10 holds great promise for the noninvasive visualization of brain tumors even when the BBB is intact, which may facilitate real-time optical image-guided brain tumor resection during surgery.

Although active targeting is valuable for delivering nanosized agents to brain tumors, it cannot guarantee $100 \%$ success in all cases. An example is the comparative study performed on liver cancer and brain cancer models 


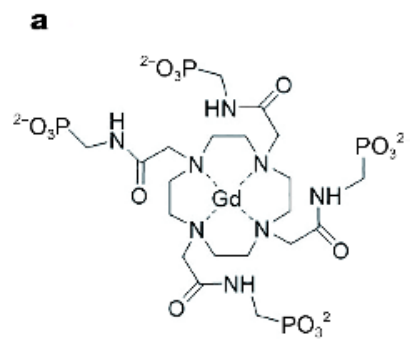

Gd-DOTA-4AmP5-

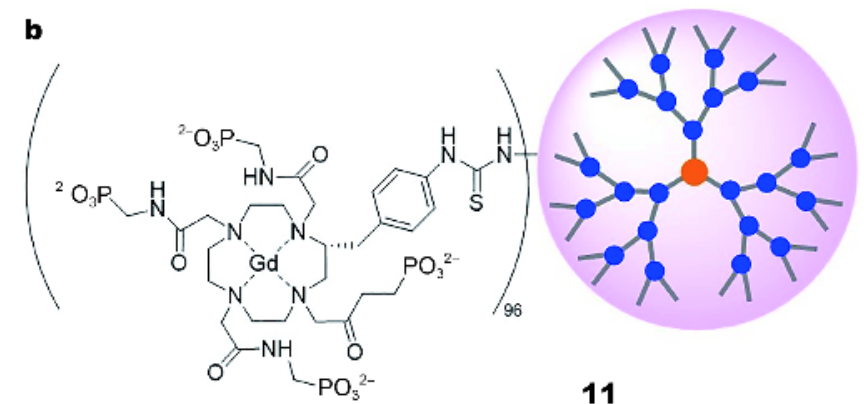

11

Figure 15 pH-sensitive MRI agents: (a) Gd-DOTA-4AmP $\mathrm{P}^{5-}$ [126] and (b) a dendrimer conjugated with Gd-DOTA-4AmP ${ }^{5-}$ at the termini [60].

using a dendrimer nanosystem to target the transferrin (Tf) receptor (TfR), which is overexpressed on cancer cells and brain cells [116]. TfR is important for the transcytosis of Tf-bound iron across brain capillary endothelial cells in humans [117], and it is highly expressed in the BBB. However, it is also expressed on hepatocytes, etc. In that comparative study, the PAMAM dendrimerbased MRI contrast agent carrying a Gd-DTPA payload was decorated with the peptide T7, a high-affinity ligand of TfR [118]. This T7-conjugated dendrimer contrast agent targeted the liver cancer more effectively than the non-targeting system [116]. However, it did not show any specificity for brain cancer. These results highlight that circumventing the BBB is a significant challenge to effective brain delivery.

\section{Stimulus-responsive targeting}

Contrast agents which are responsive to physiological conditions can expand the range of MRI beyond anatomical and functional imaging by further conveying information at the cellular or molecular level. These agents, which are referred to stimulus-responsive or smart contrast agents, are sensitive to specific fluctuations in the local environment, such as $\mathrm{pH}$, biological metal ion concentration or enzymatic activity, and are capable of reporting on such changes by inducing alterations in the MRI signal [119-124]. The change in relaxivity of these agents before and after stimulation is the most important factor in evaluating the efficiency of a stimulus-responsive contrast agent [125]. We present here stimulusresponsive dendrimer agents that are sensitive to common microenvironmental variables in vivo such as $\mathrm{pH}$, metal ions, redox potential and enzymes. These stimulusresponsive agents have the potential to offer more specific and accurate functional MRI.

\section{pH-responsive}

Cancer, inflammation and various ischemic diseases are often associated with a lower extracellular $\mathrm{pH}$, and this can be used for early detection of disease or to monitor treatment efficacy. It is therefore of great importance to non-invasively and reliably assess tissue $\mathrm{pH}$. One of the earliest and most extensively studied $\mathrm{pH}$-sensitive MRI agents is Gd-DOTA-4AmP ${ }^{5-}$ (Fig. 15a), which was developed by Sherry and coworkers [126]. The pH-sensing mechanism of this agent is based on protonation of the four phosphonate groups, which results in a 1.5-fold increase in relaxivity over the $\mathrm{pH}$ range 9.5-6.0. The phosphonate groups have $\mathrm{pK}_{\mathrm{a}}$ values in the range 6.5-8.0 and facilitate catalytic exchange of protons from the $\mathrm{Gd}^{3+}$ bound water molecule when protonated.

Sherry and colleagues [60] further reported that the relaxivity and responsiveness of Gd-DOTA-4AmP ${ }^{5-}$ was improved by conjugation to a PAMAM dendrimer (Fig. $15 \mathrm{~b})$, which generated nanoparticles with $\sim 96 \mathrm{pH}-\mathrm{re}-$ sponsive $\mathrm{Gd}^{3+}$-chelates. This $\mathrm{pH}$-responsive dendrimerbased contrast agent produced a 2.2 -fold increase in relaxivity over the $\mathrm{pH}$ range 9.5-6.0. Also, Aime and coworkers [127] investigated Gd-DOTA-functionalized poly-L-ornithine, which forms random assemblies at low $\mathrm{pH}$, but generates ordered helical structures at higher $\mathrm{pH}$. Collectively, these results demonstrate the potential for the development of effective, $\mathrm{pH}$-responsive MRI agents. It should be mentioned that the $\mathrm{pH}$ variation in most physiological or pathophysiological processes is relatively small, and may not create a large enough change in relaxivity. This may be a limitation for $\mathrm{pH}$-responsive contrast agents.

\section{Metal ion-responsive}

Metal ions are critical for many biological signaling events, redox homeostasis, and metabolism. Much effort has been dedicated to synthesizing MRI agents that respond to biologically important metal ions like $\mathrm{Ca}^{2+}, \mathrm{Zn}^{2+}$, $\mathrm{Fe}^{2+}$, and $\mathrm{Cu}^{+/ 2+}[121]$. However, only ions with concentrations above the micromolar range can be detected 




12

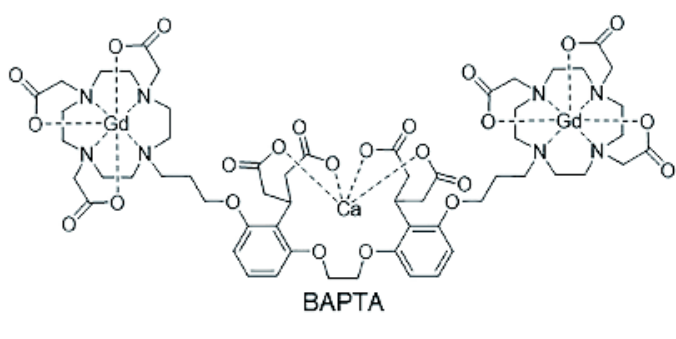

13

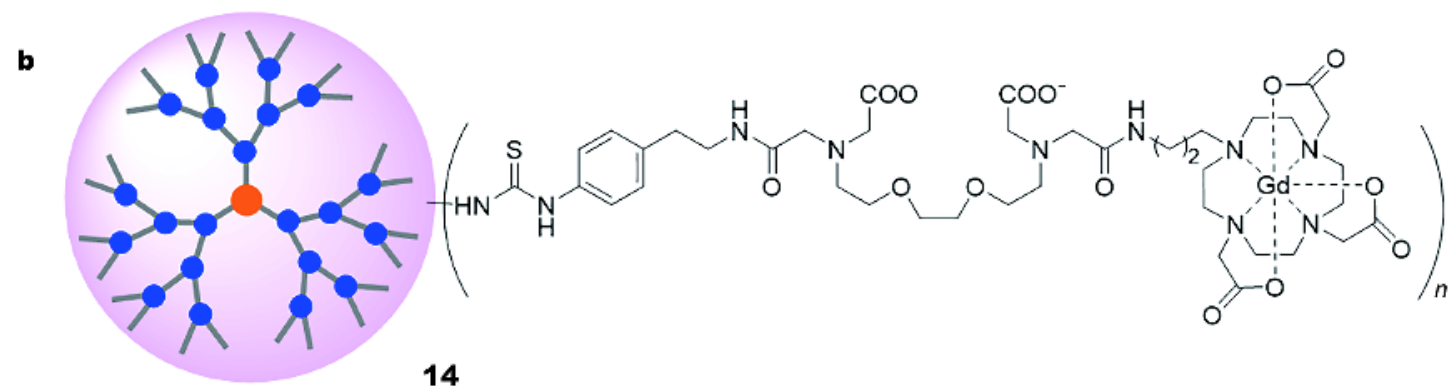

Figure 16 (a) $\mathrm{Ca}^{2+}$-sensitive MRI contrast agent developed by M eade et al. [128]; (b) dendrimer-based $\mathrm{Ca}^{2+}$-sensitive MRI contrast agent [129].

due to the low sensitivity of MRI. As $\mathrm{Ca}^{2+}$ is in high concentration and involved in neuronal signaling, several groups have developed $\mathrm{Ca}^{2+}$-responsive MRI contrast agents. Meade and colleagues [128] reported the first $\mathrm{Ca}^{2+}$-responsive agent, which consisted of two Gd-DO3A chelates joined by a modified BAPTA (1,2-bis(o-aminophenoxyl)-ethane- $N, N, N^{\prime}, N^{\prime}$-tetraacetic acid) linker (Fig. 16a). When no $\mathrm{Ca}^{2+}$ was present, the BAPTA carboxylate arms bound the chelated $\mathrm{Gd}^{3+}$, and the relaxivity was $3.26\left(\mathrm{mmol} \mathrm{L}^{-1}\right)^{-1} \mathrm{~s}^{-1}$. When $\mathrm{Ca}^{2+}$ was added, the carboxylate arms selectively bound the $\mathrm{Ca}^{2+}$, allowing the $\mathrm{Gd}^{3+}$ to bind bulk water, and increasing the relaxivity to $5.76\left(\mathrm{mmol} \mathrm{L}^{-1}\right)^{-1} \mathrm{~s}^{-1}$. Recently, Gündüz et al. [129] reported a dendrimer contrast agent that is sensitive to $\mathrm{Ca}^{2+}$ (Fig. 16b). $\mathrm{Ca}^{2+}$ was able to trigger the longitudinal relaxivity response of this agent. MRI studies in the rat cerebral cortex indicated that the diffusion properties of this agent are advantageous as compared to the monomeric equivalents.

\section{Enzyme-responsive}

Enzymatically modulated contrast agents have the potential to assess enzyme activity as well as detecting the location of enzymes and their related physiological properties. Meade et al. [130,131] developed the first examples of enzyme-sensitive contrast agents to illustrate the principle of enzymatic activation of MRI contrast agents by $\beta$-galactosidase. In addition, Chen et al. [132] developed an MRI agent based on a PAMAM dendrimer of generation 5, which carried multiple copies of the GdDOTA payload, the fluorescent dye Cy5, and the peptide ACPP, which is composed of the cell-penetrating peptide $\mathrm{Arg}_{9}$ and a gelatinase-cleavable peptide segment (Fig. 17). Gelatinases (MMP2/MMP9) belong to the matrix metalloproteinases (MMPs) and are involved in neurovascular impairment after stroke. In vivo detection of MMP2/MMP9 activity may deliver information about disruption of the $\mathrm{BBB}$, hemorrhage, and injury or death of neurons. The dendrimer agent $\mathbf{1 5}$ was used to follow the time-course of MMP2/MMP9 activity in the brain at the cellular level via a combination of fluorescent imaging and MRI. This gelatinase-specific, non-invasive agent will therefore be useful for detecting pathological processes in vivo and for validating potential treatments.

In this section, we presented dendrimer-based contrast agents which undergo changes in relaxivity according to specific fluctuations of $\mathrm{pH}$, metal ion concentration or enzymatic activity in the local environment. Together, these agents can provide valuable information on the existence and execution of numerous essential physiological and pathological processes at the cellular and molecular levels. In general, each agent is responsive and specific to a relevant physiological or pathophysiological process, and hence provides specific and more accurate 



ACPP



Figure 17 A PAMAM dendrimer of generation 5 was decorated with Gd-DOTA as an MRI reporter, Cy5 as a fluorescent dye, and the cellpenetrating peptide ACPP, which has a gelatinase-cleavable peptide segment [132].

imaging information. Nevertheless, these stimulus-responsive contrast agents have their own advantages and disadvantages. The $\mathrm{pH}$-responsive agents are less specific, as many biological events or disease lesions are associated with low $\mathrm{pH}$, such as cancer, inflammation, and ischemia. Also, most biological and pathophysiological processes result in relatively small $\mathrm{pH}$ changes, which may not create significant changes in the relaxivity of the contrast agent. The ion-responsive agents are more specific because ion-related biological processes require distinct ions, thus permitting the identification of pathophysiological events with more precision. Because of the low sensitivity of MRI, only ions with high concentrations can be detected. The most specific agents are the enzymeresponsive agents, as most enzymes have specific biological and physiological functions and activities. Nevertheless, the effectiveness of these agents may also be limited if the substrate or enzyme concentration is too low to be detected by MRI. All MRI contrast agents suffer from common limitations and drawbacks related to the low sensitivity and detection threshold of MRI. Using dendrimer platforms to load multiple copies of the responsive agent is expected to circumvent the sensitivity issue, thus enhancing the MRI signal. Moreover, different imaging modalities can be accommodated within the same dendrimer, such as $\mathbf{1 5}$ in Fig. 17, providing multimodal imaging for better and more accurate imaging.

Numerous studies have demonstrated the potential of responsive contrast agents to detect changes in diagnostically relevant variables in vitro. However, very few of these agents have been followed up in vivo. Feedback from in vivo MRI studies will be crucial for designing new and more versatile agents with structures that can be easily modified to deliver greater changes in relaxivity in response to physiological variables.

\section{Chemical exchange saturation transfer (CEST) for MRI}

As described above in the section on MRI, CEST is emerging as a novel technique [32], which enables the imaging of certain compounds at low concentrations [33]. The incorporation of metal ions results in paramagnetic CEST (PARACEST) agents, which have chemical shifts that are further away from water [32]. PARACEST agents have also been covalently linked to nanocarriers, including linear polymers, dendrimers and other macromolecules [133-136], to further improve the sensitivity of detection in vivo. For example, Ali et al. [137] conjugated the PARACEST agent Eu-DOTA-Gly ${ }_{4}$ to a PAMAM dendrimer of generation 5 (Fig. 18a), and demonstrated the CEST effect in the tumor tissue of a mouse mammary carcinoma model. Later, Ali et al. [138] created another dendrimer-based PARACEST agent by appending both Eu-DOTA-Gly ${ }_{4}$ and the fluorescent dye DL680 to the PAMAM dendrimer (Fig. 18b). This dendrimer agent exhibited effective in vivo CEST MRI in a glioblastoma mouse model, which was further validated using in vivo and ex vivo fluorescent imaging.

Recently, McMahon and co-workers [139] created dendrimers conjugated with salicylic acid as platforms for CEST. 5-Aminomethyl salicylic acid was selected as a CEST contrast agent because it has a near optimal exchange rate for detection. PAMAM dendrimers were surface-conjugated with salicylic acid, and subsequently with DOTA functions for future radiolabeling or positron emission tomography (PET)/MRI, and then the remaining amine terminals were capped with acetyl groups 

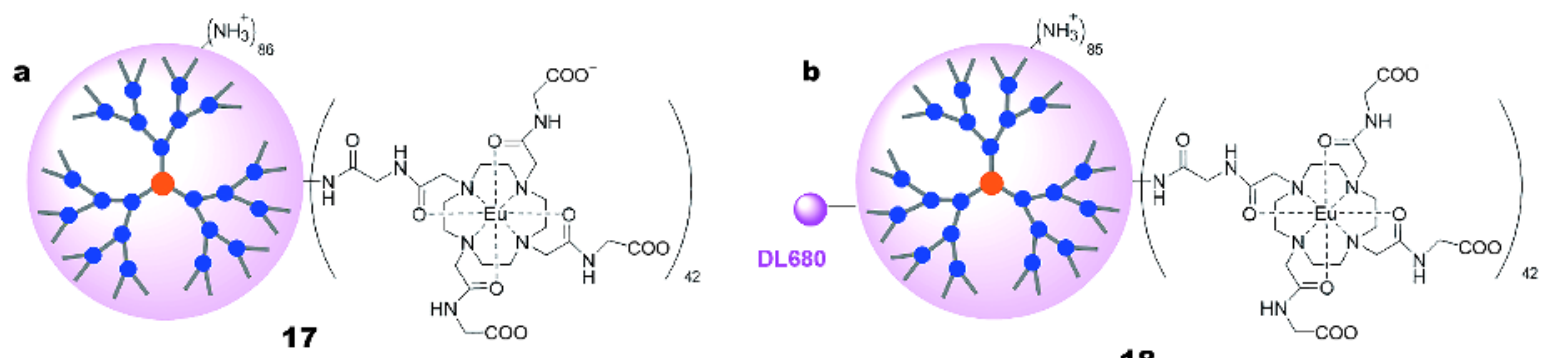

Figure 18 PAMAM dendrimers bearing (a) Eu-DOTA-Gly ${ }_{4}[137]$ and (b) both Eu-DOTA-Gly ${ }_{4}$ and the fluorescent dye DL680 [138] as PARACEST agents.

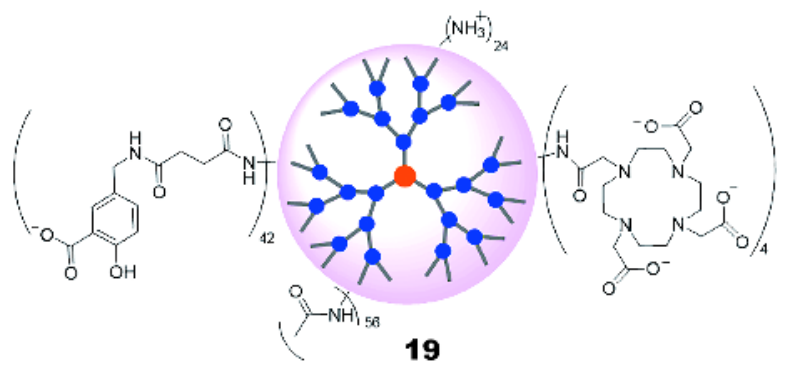

Figure 19 Salicylic acid was covalently attached to the surface of PAMAM dendrimers to generate the CEST effect in MRI [139].

(Fig. 19). The resulting agents produced strong CEST contrast at $9.4 \mathrm{ppm}$ from water with the proton exchange tunable from 1000 to $4,500 \mathrm{~s}^{-1}$, which should facilitate sensitive detection. When these agents were used to monitor convection-enhanced delivery into U87 glioblastomas in xenograft mice, the contrast persisted for more than $1.5 \mathrm{~h}$ and was distributed over $\sim 50 \%$ of the tumors [139]. This shows that salicylic acid-modified dendrimers are promising new contrast agents for medical applications.

CEST has the potential to deliver novel contrasts by using different agents based on endogenous, natural, or synthesized compounds. Importantly, for clinical applications, these agents include drugs at pharmacological concentrations. Compared to conventional MRI, CEST achieves unprecedented sensitivity at the molecular level and can potentially increase the specificity of MRI diagnoses. In addition, several PARACEST agents can be detected during a single MRI scan, which may facilitate the evaluation of multiple biomarkers. This could further increase the specificity and accuracy of MRI-based tumor detection [32].

\section{${ }^{19}$ F-MRI}

Unlike ${ }^{1} \mathrm{H}$-MRI, ${ }^{19} \mathrm{~F}$-MRI offers the unique advantage of highly sensitive imaging because of the absence of en-

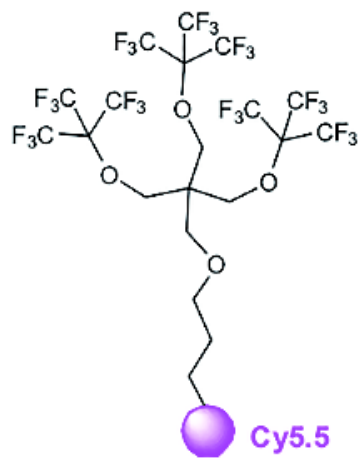

20

Figure 20 Bimodal dendrimer contrast agents bearing numerous equivalent fluorine nuclei at the dendrimer termini for ${ }^{19} \mathrm{~F}-\mathrm{MRI}$ and the near-infrared cyanine dye Cy5.5 at the dendrimer core for optical fluorescent imaging [140].

dogenous ${ }^{19} \mathrm{~F}-\mathrm{NMR}$ signals, which means that there is no background signal interference during MRI recording $[34,35]$. Also of importance is that ${ }^{19} \mathrm{~F}$ has the potential to offer quantitative MRI. Recently, Wang et al. [140] developed a bimodal dendritic imaging agent bearing numerous equivalent fluorine nuclei for ${ }^{19} \mathrm{~F}-\mathrm{MRI}$ and a near-infrared cyanine dye at the dendrimer core for optical fluorescent imaging (Fig. 20). There was a high correlation between the ${ }^{19} \mathrm{~F}-\mathrm{MRI}$ signal and the NIR fluorescence intensity in vitro and in vivo. This agent also enables quantitative ${ }^{19} \mathrm{~F}-\mathrm{MRI}$, confirmed by complementary fluorescence analysis. Consequently, dendrimer-based ${ }^{19} \mathrm{~F}$-MRI agents hold great promise for quantitative and sensitive multi-modal molecular imaging.

\section{MRI-based multimodal imaging}

Dendrimer nanoplatforms are capable of accommodating large amounts of imaging agents to enhance imaging sensitivity, and, at the same time, combining several different imaging techniques for multimodal imaging to increase the precision and reliability of diagnosis and 
treatment. Multimodal imaging agents have been created to combine MRI with other imaging techniques such as fluorescent optical imaging, computer tomography (CT), PET and single photon emission computed tomography (SPECT). Some of the dendrimer-based agents mentioned above such as 5, 9, 10, 15, 16, 18 and 20 have been used for MRI and optical fluorescent bimodal imaging, because fluorescent probes enable accurate and sensitive high-contrast cellular/tissue imaging. Bimodal imaging probes integrating the high-resolution in vivo MRI and optical fluorescence imaging into a single dendrimer platform can overcome the limitations of conventional contrast agents and facilitate quantitative MRI and fluorescence imaging. Many dendrimer-based MRI agents have therefore been developed which carry both MRI functional entities and near-infrared fluorescent dyes such as cyanine [140], rhodamine [92,93], quantum dots [141], etc, for more accurate and sensitive multi-modal imaging [41]. Often, fluorescent imaging enables the precise delineation of tumor margins and hence more complete resection of the tumor during surgery.

CT facilitates direct quantification of the contrast agent because X-ray attenuation (i.e., signal intensity) is linearly related to the concentration of the contrast agent. Regino et al. [142] reported an imaging agent based on a dendrimer of generation 8 bearing Gd-DTPA function as a dual CT and MRI contrast agent for monitoring convection-enhanced delivery to the brain. Visible enhancement of both CT and MRI was observed using this agent, and MRI offered greater sensitivity than CT. Although CT provides less anatomical contrast and lower sensitivity than MRI, the dual CT/MRI agent can act as a surrogate marker for convection-enhanced delivery of macromolecular therapeutic agents to the brain.

PET and SPECT can deliver functional information with high sensitivity. However, both of them are limited by low anatomical resolution. MRI offers high-resolution anatomical images, particularly when a specific contrast agent is used. Therefore, PET and SPECT in combination with MRI have been integrated within the dendrimer platform and applied to improve the precision and accuracy of imaging and diagnosis [41]. For example, Luo et al. [143] developed a dendrimer platform carrying DOTA functionalities to chelate $\mathrm{Mn}^{2+}$ for $\mathrm{MRI}$ and ${ }^{99 \mathrm{~m}} \mathrm{Tc}$ for SPECT imaging along with folic acid as the ligand for targeted tumor imaging. The success of the multimodal imaging approach may permit earlier and more accurate detection of tumors, as well as providing further insights into the molecular mechanisms underlying cancer development.

\section{MRI-based theranostics}

Besides carrying several probes for multimodal imaging, dendrimers can also combine imaging and therapeutic agents within the same nanoparticle to create theranostic platforms. Yang et al. [144] reported a PAMAM dendrimer covalently linked to gadolinium-functionalized nanographene oxide (Gd-NGO). The aim of this nanosystem was to deliver chemotherapeutic drugs and genetargeting microRNAs (miRNAs) to cancer cells, while also carrying an MRI contrast agent to identify the location and extent of BBB openings and quantitate drug delivery to tumor tissues. The positively charged surface of the dendrimer-Gd-NGO conjugate simultaneously adsorbed the anticancer drug epirubicin (EPI) and bound to the miRNA Let-7g (Fig. 21). The so-constructed theranostic agent $\mathbf{2 1}$ had considerably higher transfection efficiency than nanoparticles loaded with only EPI or miRNA, and also more strongly inhibited the growth of cancer cells in human glioblastoma (U87) models. These findings indicate that $\mathbf{2 1}$ may be a promising non-viral vector for combining chemotherapy, miRNA delivery and molecular imaging diagnosis in clinical applications.

\section{CONCLUSION AND PERSPECTIVES}

Since the first clinical contrast agent Gd-DPTA was ap-

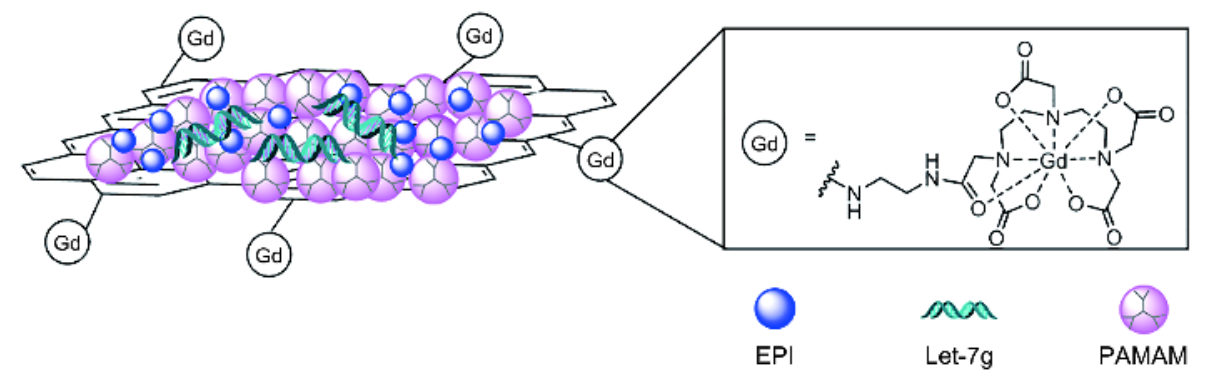

Figure 21 PAMAM dendrimer conjugated with gadolinium-functionalized nanographene oxide (Gd-NGO) entraps the anticancer drug epirubicin (EPI) and interacts with the negatively charged miRNA Let-7g as a theranostic nanosystem [144]. 
proved in 1988, low molecular weight agents have been crucial for improving MRI contrast. Nevertheless, macromolecular agents have been added to the arsenal in order to address the limitations of these low molecular weight agents, i.e., fast renal clearance, non-specificity and low imaging efficiency. Dendrimers, a special family of synthetic macromolecules, have generated tremendous interest as core platforms for constructing MRI agents. Dendrimers have tunable chemistry, which permits a precise "chemical makeup", and can carry a high payload within a nanosized three-dimensional space. The size and shape of the dendrimer, as well as the surface distribution of the desired functionality, can be adjusted to enhance the imaging sensitivity and impart targeted imaging. By virtue of their large molecular weight and steric hindrance, dendrimer agents have greater relaxivity than low molecular weight agents such as Gd-DPTA and GdDOTA. In addition, the large number of modifiable terminals and the possibility of precise chemical decoration with multiple modifications and/or target molecules, permits dendrimers to act as effective and specific agents. Surface modification includes targeting moieties for precise and sensitive molecular imaging and PEGylation to improve circulation half-lives and biodistribution. Also, combinations of different imaging techniques for multimodal imaging or integration of therapeutic and imaging modalities as theranostics within a single dendrimer can be easily realized and have been applied to brain cancer.

As previously discussed, brain cancer is particularly challenging to manage because the BBB impedes the delivery of therapeutic and imaging agents for treatment and diagnosis. Luckily, the BBB is often disrupted by the uncontrolled cancer cell proliferation and the abnormal angiogenesis in the tumor, resulting in leaky tumor blood vessels, and hence the EPR effect. These features have been exploited to develop nanosized agents for therapeutic and imaging purposes. In this vein, dendrimers with precise nanostructure and cooperative multivalence yet differing in core, branching, generation and surface composition have been investigated as MRI agents for brain cancer, and some of them show promise. To further improve the imaging quality, active targeting has been integrated into dendrimer platforms to circumvent the BBB (both compromised and intact) as well as specifically targeting brain cancer. Although targeted agents have proved to be superior to non-targeted systems in general, there is no guaranteed success, highlighting the challenge of overcoming the BBB for effective brain delivery. Dendrimer contrast agents with specific functionalities to respond to $\mathrm{pH}$ change, metal ions and enzyme activity have also been explored for molecular imaging. In addition, CEST and PARACEST agents have been further developed to improve imaging specificity and sensitivity.

It is worth noting that although most dendrimer contrast agents have been constructed using commercially available dendrimers such as PAMAM, PPI and polylysine dendrimers, the potential diversity of the dendrimer core, the branching units, and the internal and surface functionalities is immense for creating dendrimer-based MRI contrast agents, multimodal imaging agents or theranostics. In addition, most investigations into dendrimer contrast agents have been performed in the context of preclinical imaging studies in animal models. Translation of the results and knowledge obtained from these model systems to the clinic lies a long way ahead. Regulatory agencies also face a number of challenging issues, such as consistent characterization and reproducible production, which are required for the realworld implementation of contrast agents with outstanding properties. Feedback from biologists and clinicians to chemists will be essential for establishing new and more effective agents which can deliver the required imaging precision and quality.

Received 23 May 2018; accepted 11 July 2018;

published online 31 July 2018

1 Noone AM, Howlader N, Krapcho M, et al. (eds.). SEER Cancer Statistics Review, 1975-2013. National Cancer Institute, 2016

2 Siegel R, Naishadham D, Jemal A. Cancer statistics, 2012. CA-A Cancer J Clin, 2012, 62: 10-29

3 Preusser M, Marosi C. Advances in brain tumour classification and therapy. Nat Rev Neurol, 2016, 13: 71-72

4 Louis D, Ohgaki $\mathrm{H}$, Wiestler O, et al. WHO Classification of Tumours of the Central Nervous System ( $4^{\text {th }}$ ed.). Lyon: WHO/ IARC., 2007

5 Davis ME. Glioblastoma: overview of disease and treatment. Clin J Oncol Nurs, 2016, 20: S2-S8

6 Bloch O, Han SJ, Cha S, et al. Impact of extent of resection for recurrent glioblastoma on overall survival. J Neurosurgery, 2012, 117: $1032-1038$

7 Barone DG, Lawrie TA, Hart MG. Image guided surgery for the resection of brain tumours. Cochrane Database Systatic Rev, 2014, CD009685

8 Abbott NJ, Patabendige AAK, Dolman DEM, et al. Structure and function of the blood-brain barrier. NeuroBiol Dis, 2010, 37: 1325

9 Obermeier B, Daneman R, Ransohoff RM. Development, maintenance and disruption of the blood-brain barrier. Nat Med, 2013, 19: 1584-1596

10 Banks WA. From blood-brain barrier to blood-brain interface: new opportunities for CNS drug delivery. Nat Rev Drug Discov, 2016, 15: 275-292

11 Deeken JF, Löscher W. The blood-brain barrier and cancer: transporters, treatment, and Trojan horses. Clinical Cancer Res, 
2007, 13: 1663-1674

12 Cuddapah VA, Robel S, Watkins S, et al. A neurocentric perspective on glioma invasion. Nat Rev Neurosci, 2014, 15: 455-465

13 Matsumura Y, Maeda H. A new concept for macromolecular therapeutics in cancer chemotherapy: mechanism of tumoritropic accumulation of proteins and the antitumor agent smancs. Cancer Res, 1986, 46: 6387-6392

14 Maeda H, Nakamura H, Fang J. The EPR effect for macromolecular drug delivery to solid tumors: Improvement of tumor uptake, lowering of systemic toxicity, and distinct tumor imaging in vivo. Adv Drug Deliver Rev, 2013, 65: 71-79

15 Maeda H. Toward a full understanding of the EPR effect in primary and metastatic tumors as well as issues related to its heterogeneity. Adv Drug Deliver Rev, 2015, 91: 3-6

16 Mormina E, Petracca M, Bommarito G, et al. Cerebellum and neurodegenerative diseases: Beyond conventional magnetic resonance imaging. WJR, 2017, 9: 371-388

17 van der Kolk AG, Hendrikse J, Zwanenburg JJM, et al. Clinical applications of 7T MRI in the brain. Eur J Rad, 2013, 82: 708-718

18 Vargas MI, Martelli P, Xin L, et al. Clinical neuroimaging using 7 T MRI: challenges and prospects. J NeuroImag, 2018, 28: 5-13

19 Terreno E, Castelli DD, Viale A, et al. Challenges for molecular magnetic resonance imaging. Chem Rev, 2010, 110: 3019-3042

20 Villanueva-Meyer JE, Mabray MC, Cha S. Current clinical brain tumor imaging. Neurosurgery, 2017, 81: 397-415

21 Merbach A S, Helm L, Toth E (eds.). The Chemistry of Contrast Agents in Medical Magnetic Resonance Imaging. West Sussex: John Wiley \& Sons, 2013

22 Geraldes CFGC, Laurent S. Classification and basic properties of contrast agents for magnetic resonance imaging. Contrast Media Mol Imag, 2009, 4: 1-23

23 Evans CH, Biochemistry of the Lanthanides. New York: Springer Science \& Business Media, 2013

24 Bellin MF. MR contrast agents, the old and the new. Eur J Rad, 2006, 60: 314-323

25 Ersoy H, Rybicki FJ. Biochemical safety profiles of gadoliniumbased extracellular contrast agents and nephrogenic systemic fibrosis. J Magn Reson Imag, 2007, 26: 1190-1197

26 Chang CA. Magnetic resonance imaging contrast agents design and physicochemical properties of gadodiamide. Invest Radiol, 1993, 28: S21-S27

27 Aime S, Botta M, Ermondi G. NMR study of solution structures and dynamics of lanthanide(III) complexes of DOTA. Inorg Chem, 1992, 31: 4291-4299

28 Caravan P, Ellison JJ, McMurry TJ, et al. Gadolinium(III) chelates as MRI contrast agents: structure, dynamics, and applications. Chem Rev, 1999, 99: 2293-2352

29 Hermann P, Kotek J, Kubícek V, et al. Gadolinium(III) complexes as MRI contrast agents: ligand design and properties of the complexes. Dalton Trans, 2008, 14: 3027

30 Zhou Z, Lu ZR. Gadolinium-based contrast agents for magnetic resonance cancer imaging. WIREs Nanomed Nanobiotechnol, 2013, 5: 1-18

31 Lauterbur P, Mendoca-Dias M, Rudin AM. Augmentation of tissue water proton spin-lattice relaxation rates by in vivo addition of paramagnetic ions. In: Dutton PL, Leigh JS, Scarpa A (eds.). Frontier of Biological Energetics. New York: Academic Press, 1978, 752-759

32 Zhang S, Merritt M, Woessner DE, et al. PARACEST agents: modulating MRI contrast via water proton exchange. Acc Chem
Res, 2003, 36: 783-790

33 Wu B, Warnock G, Zaiss M, et al. An overview of CEST MRI for non-MR physicists. EJNMMI Phys, 2016, 3: 19

34 Tirotta I, Dichiarante V, Pigliacelli C, et al. ${ }^{19} \mathrm{~F}$ magnetic resonance imaging (MRI): from design of materials to clinical applications. Chem Rev, 2014, 115: 1106-1129

35 Chen H, Viel S, Ziarelli F, et al. ${ }^{19} \mathrm{~F}$ NMR: a valuable tool for studying biological events. Chem Soc Rev, 2013, 42: 7971-7982

36 L. Villaraza AJ, Bumb A, Brechbiel MW. Macromolecules, dendrimers, and nanomaterials in magnetic resonance imaging: the interplay between size, function, and pharmacokinetics. Chem Rev, 2010, 110: 2921-2959

37 Venditto VJ, Regino CAS, Brechbiel MW. PAMAM dendrimer based macromolecules as improved contrast agents. Mol Pharm, 2005, 2: 302-311

$38 \mathrm{Ni} \mathrm{D}, \mathrm{Bu}$ W, Ehlerding EB, et al. Engineering of inorganic nanoparticles as magnetic resonance imaging contrast agents. Chem Soc Rev, 2017, 46: 7438-7468

39 Shin TH, Choi Y, Kim S, et al. Recent advances in magnetic nanoparticle-based multi-modal imaging. Chem Soc Rev, 2015, 44: 4501-4516

40 Langereis S, Dirksen A, Hackeng TM, et al. Dendrimers and magnetic resonance imaging. New J Chem, 2007, 31: 1152-1160

41 Qiao Z, Shi X. Dendrimer-based molecular imaging contrast agents. Prog Polymer Sci, 2015, 44: 1-27

42 McMahon MT, Bulte JWM. Two decades of dendrimers as versatile MRI agents: a tale with and without metals. WIREs Nanomed Nanobiotechnol, 2018, 10: e1496

43 Tomalia DA, Naylor AM, Goddard WA. Starburst dendrimers: molecular-level control of size, shape, surface chemistry, topology, and flexibility from atoms to macroscopic matter. Angew Chem Int Ed, 1990, 29: 138-175

44 Tomalia DA, Fréchet JMJ. Discovery of dendrimers and dendritic polymers: A brief historical perspective. J Polym Sci A Polym Chem, 2002, 40: 2719-2728

45 Vögtle F, Richardt G, Werner N. Dendrimer Chemistry: Concepts, Syntheses, Properties, Applications. Weinheim: John Wiley \& Sons, 2009

46 Tomalia DA, Christensen JB, Boas U. Dendrimers, Dendrons, and Dendritic Polymers: Discovery, Applications, and the Future. Cambridge: Cambridge University Press, 2012

47 Walter MV, Malkoch M. Simplifying the synthesis of dendrimers: accelerated approaches. Chem Soc Rev, 2012, 41: 4593-4609

48 Grayson SM, Fréchet JMJ. Convergent dendrons and dendrimers: from synthesis to applications. Chem Rev, 2001, 101: 3819-3868

49 Franc G, Kakkar AK. "Click" methodologies: efficient, simple and greener routes to design dendrimers. Chem Soc Rev, 2010, 39: 1536-1544

50 Arseneault M, Wafer C, Morin JF. Recent advances in click chemistry applied to dendrimer synthesis. Molecules, 2015, 20: 9263-9294

51 Lee CC, MacKay JA, Fréchet JMJ, et al. Designing dendrimers for biological applications. Nat Biotechnol, 2005, 23: 1517-1526

52 Mintzer MA, Grinstaff MW. Biomedical applications of dendrimers: a tutorial. Chem Soc Rev, 2011, 40: 173-190

53 Wiener E, Brechbiel MW, Brothers H, et al. Dendrimer-based metal chelates: A new class of magnetic resonance imaging contrast agents. Magn Reson Med, 1994, 31: 1-8

54 Margerum LD, Campion BK, Koo M, et al. Gadolinium(III) DO3A macrocycles and polyethylene glycol coupled to den- 
drimers effect of molecular weight on physical and biological properties of macromolecular magnetic resonance imaging contrast agents. J Alloys Compd, 1997, 249: 185-190

55 Bryant Jr LH, Brechbiel $\mathrm{MW}, \mathrm{Wu} \mathrm{C}$, et al. Synthesis and relaxometry of high-generation $(G=5,7,9$, and 10) PAMAM dendrimer-DOTA-gadolinium chelates. J Magn Reson Imaging, 1999, 9: 348-352

56 Langereis S, de Lussanet QG, van Genderen MHP, et al. Multivalent contrast agents based on gadolinium-diethylenetriaminepentaacetic acid-terminated poly(propylene imine) dendrimers for magnetic resonance imaging. Macromolecules, 2004, 37: 3084-3091

57 Tóth É, Pubanz D, Vauthey S, et al. The role of water exchange in attaining maximum relaxivities for dendrimeric MRI contrast agents. Chem Eur J, 1996, 2: 1607-1615

58 Laus S, Sour A, Ruloff R, et al. Rotational dynamics account for $\mathrm{pH}$-dependent relaxivities of PAMAM dendrimeric, Gd-based potential MRI contrast agents. Chem Eur J, 2005, 11: 3064-3076

59 Laus S, Ruloff R, Tóth E, et al. $\mathrm{Gd}^{\mathrm{III}}$ complexes with fast water exchange and high thermodynamic stability: potential building blocks for high-relaxivity MRI contrast agents. Chem Eur J, 2003, 9: 3555-3566

60 Ali MM, Woods M, Caravan P, et al. Synthesis and relaxometric studies of a dendrimer-based $\mathrm{pH}$-responsive MRI contrast agent. Chem Eur J, 2008, 14: 7250-7258

61 Kobayashi H, Kawamoto S, Star RA, et al. Activated clearance of a biotinylated macromolecular MRI contrast agent from the blood pool using an avidin chase. Bioconjugate Chem, 2003, 14: 10441047

62 Kobayashi H, Kawamoto S, Star RA, et al. Micro-magnetic resonance lymphangiography in mice using a novel dendrimerbased magnetic resonance imaging contrast agent. Cancer Res, 2003, 63: 271-276

63 Yordanov AT, Kobayashi H, English SJ, et al. Gadolinium-labeled dendrimers as biometric nanoprobes to detect vascular permeability. J Mater Chem, 2003, 13: 1523-1525

64 Langereis S, de Lussanet QG, van Genderen MHP, et al. Evaluation of Gd(III)DTPA-terminated poly(propylene imine) dendrimers as contrast agents for MR imaging. NMR Biomed, 2006, 19: 133-141

65 Stasko NA, Johnson CB, Schoenfisch MH, et al. Cytotoxicity of polypropylenimine dendrimer conjugates on cultured endothelial cells. Biomacromolecules, 2007, 8: 3853-3859

66 Mishra V, Gupta U, Jain N. Influence of different generations of poly(propylene imine) dendrimers on human erythrocytes. Die Pharmazie, 2010, 65: 891-895

67 Kobayashi H, Brechbiel MW. Nano-sized MRI contrast agents with dendrimer cores. Adv Drug Deliver Rev, 2005, 57: 22712286

68 Weinmann H, Ebert W, Wagner S, et al. MR angio with special focus on blood-pool agents, In Proceedings of the IX International Workshop on Magnetic Resonance Angiography, Valencia, 1997, pp 335-340

69 Herborn CU, Barkhausen J, Paetsch I, et al. Coronary arteries: contrast-enhanced MR imaging with SH L 643A-experience in 12 volunteers. Radiology, 2003, 229: 217-223

70 Herborn CU, Schmidt M, Bruder O, et al. MR coronary angiography with SH L 643 A: initial experience in patients with coronary artery disease. Radiology, 2004, 233: 567-573

71 Su MY, Mühler A, Lao X, et al. Tumor characterization with dynamic contrast-enhanced MRI using MR contrast agents of various molecular weights. Magn Reson Med, 1998, 39: 259-269

72 Roberts HC, Saeed M, Roberts TP, et al. MRI of acute myocardial ischemia: Comparing a new contrast agent, Gd-DTPA-24-cascade-polymer, with Gd-DTPA. J Magn Reson Imaging, 1999, 9: 204-208

73 Shi J, Kantoff PW, Wooster R, et al. Cancer nanomedicine: progress, challenges and opportunities. Nat Rev Cancer, 2017, 17: $20-37$

74 Gupta B, Levchenko TS, Torchilin VP. TAT peptide-modified liposomes provide enhanced gene delivery to intracranial human brain tumor xenografts in nude mice. Oncol Res, 2006, 16: 351359

75 Oller-Salvia B, Sánchez-Navarro M, Giralt E, et al. Blood-brain barrier shuttle peptides: an emerging paradigm for brain delivery. Chem Soc Rev, 2016, 45: 4690-4707

76 Hervé F, Ghinea N, Scherrmann JM. CNS delivery via adsorptive transcytosis. AAPS J, 2008, 10: 455-472

77 Huang R, Ke W, Liu Y, et al. The use of lactoferrin as a ligand for targeting the polyamidoamine-based gene delivery system to the brain. Biomaterials, 2008, 29: 238-246

78 Kumar $\mathrm{P}, \mathrm{Wu} \mathrm{H}, \mathrm{McBride} \mathrm{JL}$, et al. Transvascular delivery of small interfering RNA to the central nervous system. Nature, 2007, 448: 39-43

79 Zhan C, Li B, Hu L, et al. Micelle-based brain-targeted drug delivery enabled by a nicotine acetylcholine receptor ligand. Angew Chem Int Ed, 2011, 50: 5482-5485

80 Bertrand N, Wu J, Xu X, et al. Cancer nanotechnology: the impact of passive and active targeting in the era of modern cancer biology. Adv Drug Deliver Rev, 2014, 66: 2-25

81 Dai L, Liu J, Luo Z, et al. Tumor therapy: targeted drug delivery systems. J Mater Chem B, 2016, 4: 6758-6772

82 Davis ME, Zuckerman JE, Choi CHJ, et al. Evidence of RNAi in humans from systemically administered siRNA via targeted nanoparticles. Nature, 2010, 464: 1067-1070

83 Sugahara KN, Teesalu T, Karmali PP, et al. Coadministration of a tumor-penetrating peptide enhances the efficacy of cancer drugs. Science, 2010, 328: 1031-1035

84 Li M, Luo Z, Xia Z, et al. Time-sequenced drug delivery approaches towards effective chemotherapeutic treatment of glioma. Mater Horiz, 2017, 4: 977-996

85 Lajoie JM, Shusta EV. Targeting receptor-mediated transport for delivery of biologics across the blood-brain barrier. Annu Rev Pharmacol Toxicol, 2015, 55: 613-631

86 Cheng Y, Morshed RA, Auffinger B, et al. Multifunctional nanoparticles for brain tumor imaging and therapy. Adv Drug Deliver Rev, 2014, 66: 42-57

87 Mishra V, Kesharwani P. Dendrimer technologies for brain tumor. Drug Discovery Today, 2016, 21: 766-778

$88 \mathrm{Xu} \mathrm{H}$, Regino CAS, Bernardo $\mathrm{M}$, et al. Toward improved syntheses of dendrimer-based magnetic resonance imaging contrast agents: new bifunctional diethylenetriaminepentaacetic acid ligands and nonaqueous conjugation chemistry. J Med Chem, 2007, 50: 3185-3193

89 Karki K, Ewing JR, Ali MM. Targeting glioma with a dual mode optical and paramagnetic nanoprobe across the blood-brain tumor barrier. J Nanomed Nanotechnol, 2016, 7: 395

90 Jayasundara S, Ali MM. Dendrimer-based Nanoparticles for Targeted Brain Tumor Imaging. J Nanomed Nanotechnol, 2017, 08: 444 
91 Nagy JA, Chang SH, Dvorak AM, et al. Why are tumour blood vessels abnormal and why is it important to know? Br J Cancer, 2009, 100: 865-869

92 Sarin H, Kanevsky AS, Wu H, et al. Effective transvascular delivery of nanoparticles across the blood-brain tumor barrier into malignant glioma cells. J Transl Med, 2008, 6: 80

93 Sarin H, Kanevsky AS, Wu H, et al. Physiologic upper limit of pore size in the blood-tumor barrier of malignant solid tumors. J Transl Med, 2009, 7: 51

94 Chauhan VP, Stylianopoulos T, Martin JD, et al. Normalization of tumour blood vessels improves the delivery of nanomedicines in a size-dependent manner. Nat Nanotech, 2012, 7: 383-388

95 Chauhan VP, Jain RK. Strategies for advancing cancer nanomedicine. Nat Mater, 2013, 12: 958-962

96 Alamdari NH, Alaei-Beirami M, Shandiz SAS, et al. Gd ${ }^{3+}$-asparagine-anionic linear globular dendrimer second-generation G2 complexes: novel nanobiohybrid theranostics. Contrast Media Mol Imaging, 2017, 3625729

97 Fulton DA, O'Halloran M, Parker D, et al. Efficient relaxivity enhancement in dendritic gadolinium complexes: effective motional coupling in medium molecular weight conjugates. Chem Commun, 2005, 474

98 Takahashi M, Hara Y, Aoshima K, et al. Utilization of dendritic framework as a multivalent ligand: a functionalized gadolinium (III) carrier with glycoside cluster periphery. Tetrahedron Lett, 2000, 41: 8485-8488

99 Bertin A, Steibel J, Michou-Gallani AI, et al. Development of a dendritic manganese-enhanced magnetic resonance imaging (MEMRI) contrast agent: synthesis, toxicity (in vitro) and relaxivity (in vitro, in vivo) studies. Bioconjugate Chem, 2009, 20: 760767

100 Nakamura Y, Mochida A, Choyke PL, et al. Nanodrug delivery: is the enhanced permeability and retention effect sufficient for curing cancer? Bioconjugate Chem, 2016, 27: 2225-2238

101 Lyons SA, O'Neal J, Sontheimer H. Chlorotoxin, a scorpion-derived peptide, specifically binds to gliomas and tumors of neuroectodermal origin. Glia, 2002, 39: 162-173

102 Veiseh M, Gabikian P, Bahrami SB, et al. Tumor paint: a chlorotoxin:Cy5.5 bioconjugate for intraoperative visualization of cancer foci. Cancer Res, 2007, 67: 6882-6888

103 Mamelak AN, Jacoby DB. Targeted delivery of antitumoral therapy to glioma and other malignancies with synthetic chlorotoxin (TM-601). Expert Opin Drug Deliver, 2007, 4: 175-186

104 Huang $\mathrm{R}$, Han L, Li J, et al. Chlorotoxin-modified macromolecular contrast agent for MRI tumor diagnosis. Biomaterials, 2011, 32: 5177-5186

105 Shibata M, Yamada S, Kumar SR, et al. Clearance of Alzheimer's amyloid- $\beta 1-40$ peptide from brain by LDL receptor-related protein-1 at the blood-brain barrier. J Clin Invest, 2000, 106: 1489-1499

106 Maletinska L, Blakely EA, Bjornstad KA, et al. Human glioblastoma cell lines: Levels of low-density lipoprotein receptor and low-density lipoprotein receptor-related protein. Cancer Res, 2000, 60: 2300-2303

107 Demeule M, Régina A, Ché C, et al. Identification and design of peptides as a new drug delivery system for the brain. J Pharmacol Exp Therapeutics, 2008, 324: 1064-1072

108 Drappatz J, Brenner A, Wong ET, et al. Phase I study of GRN1005 in recurrent malignant glioma. Clin Cancer Res, 2013, 19: 15671576
109 Yan H, Wang J, Yi P, et al. Imaging brain tumor by dendrimerbased optical/paramagnetic nanoprobe across the blood-brain barrier. Chem Commun, 2011, 47: 8130-8132

110 Yan H, Wang L, Wang J, et al. Two-order targeted brain tumor imaging by using an optical/paramagnetic nanoprobe across the blood brain barrier. ACS Nano, 2012, 6: 410-420

111 Brooks PC, Clark RAF, Cheresh DA. Requirement of vascular integrin $\alpha_{v} \beta_{3}$ for angiogenesis. Science, 1994, 264: 569-571

112 Hynes RO. Integrins. Cell, 2002, 110: 673-687

113 Cui Q, Yang S, Ye P, et al. Downregulation of TLX induces TET3 expression and inhibits glioblastoma stem cell self-renewal and tumorigenesis. Nat Commun, 2016, 7: 10637

114 Liu X, Liu C, Chen C, et al. Targeted delivery of dicer-substrate siRNAs using a dual targeting peptide decorated dendrimer delivery system. NanoMed-Nanotechnol Biol Med, 2014, 10: 16271636

115 Schottelius M, Laufer B, Kessler $\mathrm{H}$, et al. Ligands for mapping $\alpha_{v} \beta_{3}$-integrin expression in vivo. Acc Chem Res, 2009, 42: 969980

116 Han L, Li J, Huang S, et al. Peptide-conjugated polyamidoamine dendrimer as a nanoscale tumor-targeted T1 magnetic resonance imaging contrast agent. Biomaterials, 2011, 32: 2989-2998

117 Pardridge WM, Eisenberg J, Yang J. Human blood-brain barrier transferrin receptor. Metabolism, 1987, 36: 892-895

118 Lee JH, Engler JA, Collawn JF, et al. Receptor mediated uptake of peptides that bind the human transferrin receptor. Eur J Biochem, 2001, 268: 2004-2012

$119 \mathrm{Tu}$ C, Osborne EA, Louie AY. Activatable T1 and T2 magnetic resonance imaging contrast agents. Ann Biomed Eng, 2011, 39: $1335-1348$

120 De Leon-Rodriguez LM, Lubag AJM, Malloy CR, et al. Responsive MRI agents for sensing metabolism in vivo. Acc Chem Res, 2009, 42: 948-957

121 Que EL, Chang CJ. Responsive magnetic resonance imaging contrast agents as chemical sensors for metals in biology and medicine. Chem Soc Rev, 2010, 39: 51-60

122 Heffern MC, Matosziuk LM, Meade TJ. Lanthanide probes for bioresponsive imaging. Chem Rev, 2013, 114: 4496-4539

123 Angelovski G. What we can really do with bioresponsive MRI contrast agents. Angew Chem Int Ed, 2016, 55: 7038-7046

124 Angelovski G. Heading toward macromolecular and nanosized bioresponsive MRI probes for successful functional imaging. Acc Chem Res, 2017, 50: 2215-2224

125 Caravan P. Strategies for increasing the sensitivity of gadolinium based MRI contrast agents. Chem Soc Rev, 2006, 35: 512-523

126 Zhang $\mathrm{S}$, Wu K, Sherry AD. A novel $\mathrm{pH}$-sensitive MRI contrast agent. Angew Chem Int Ed, 1999, 38: 3192-3194

127 Aime $\mathrm{S}$, Fedeli $\mathrm{F}$, Sanino A, et al. $\mathrm{A}_{2} / \mathrm{R}_{1}$ ratiometric procedure for a concentration-independent, $\mathrm{pH}$-responsive, $\mathrm{Gd}(\mathrm{III})$-based MRI agent. J Am Chem Soc, 2006, 128: 11326-11327

128 Li W, Fraser SE, Meade TJ. A calcium-sensitive magnetic resonance imaging contrast agent. J Am Chem Soc, 1999, 121: 1413-1414

129 Gündüz S, Nitta N, Vibhute S, et al. Dendrimeric calcium-responsive MRI contrast agents with slow in vivo diffusion. Chem Commun, 2015, 51: 2782-2785

130 Louie AY, Hüber MM, Ahrens ET, et al. In vivo visualization of gene expression using magnetic resonance imaging. Nat Biotechnol, 2000, 18: 321-325

131 Moats RA, Fraser SE, Meade TJ. A "smart" magnetic resonance 
imaging agent that reports on specific enzymatic activity. Angew Chem Int Ed Engl, 1997, 36: 726-728

132 Chen S, Cui J, Jiang T, et al. Gelatinase activity imaged by activatable cell-penetrating peptides in cell-based and in vivo models of stroke. J Cereb Blood Flow Metab, 2017, 37: 188-200

133 Pikkemaat JA, Wegh RT, Lamerichs R, et al. Dendritic PARACEST contrast agents for magnetic resonance imaging. Contrast Media Mol Imaging, 2007, 2: 229-239

134 Wu Y, Zhou Y, Ouari O, et al. Polymeric PARACEST agents for enhancing MRI contrast sensitivity. J Am Chem Soc, 2008, 130: 13854-13855

135 Vasalatiy O, Gerard RD, Zhao P, et al. Labeling of adenovirus particles with PARACEST agents. Bioconjugate Chem, 2008, 19: 598-606

136 Zhang S, Winter P, Wu K, et al. A novel europium(III)-based MRI contrast agent. J Am Chem Soc, 2001, 123: 1517-1518

137 Ali MM, Yoo B, Pagel MD. Tracking the relative in vivo pharmacokinetics of nanoparticles with PARACEST MRI. Mol Pharm, 2009, 6: 1409-1416

138 Ali MM, Bhuiyan MP, Janic B, et al. A nano-sized PARACESTfluorescence imaging contrast agent facilitates and validates in vivo CEST MRI detection of glioma. Nanomedicine, 2012, 7: 1827-1837

139 Lesniak WG, Oskolkov N, Song X, et al. Salicylic acid conjugated dendrimers are a tunable, high performance CEST MRI NanoPlatform. Nano Lett, 2016, 16: 2248-2253

140 Wang Z, Yue X, Wang Y, et al. A symmetrical fluorous dendroncyanine dye-conjugated bimodal nanoprobe for quantitative ${ }^{19} \mathrm{~F}$ MRI and NIR fluorescence bioimaging. Adv Healthcare Mater,
2014, 3: 1326-1333

141 Bakalova R, Zhelev Z, Kokuryo D, et al. Chemical nature and structure of organic coating of quantum dots is crucial for their application in imaging diagnostics. Int J Nanomedicine, 2011, 6: 1719-1732

142 Regino CAS, Walbridge S, Bernardo M, et al. A dual CT-MR dendrimer contrast agent as a surrogate marker for convectionenhanced delivery of intracerebral macromolecular therapeutic agents. Contrast Media Mol Imaging, 2008, 3: 2-8

143 Luo Y, Zhao L, Li X, et al. The design of a multifunctional dendrimer-based nanoplatform for targeted dual mode SPECT/MR imaging of tumors. J Mater Chem B, 2016, 4: 7220-7225

144 Yang HW, Huang CY, Lin CW, et al. Gadolinium-functionalized nanographene oxide for combined drug and microRNA delivery and magnetic resonance imaging. Biomaterials, 2014, 35: 65346542

Acknowledgements Financial support from La Ligue Nationale Contre le Cancer (EL2016.LNCC/LPP to Peng L, PhD fellowship to Lyu $\mathrm{Z})$, the French National Research Agency under the frame of EuroNanoMed II (ANR-15-ENM2-0006-02, ANR-16-ENM2-0004-02) (Peng L), the Campus France ORCHID program (Peng L, Kao CL) and China Scholarship Council (Ding L) is gratefully acknowledged.

Author contributions Peng L coordinated the project, and all authors contributed to and approved the manuscript.

Conflict of interest The authors declare that they have no conflict of interest. 




Ling Ding is a PhD student under the co-direction of Drs Monique Bernard and Ling Peng at Aix-Marseille University in France. Her PhD project is mainly focused on elaborating dendrimer nanosystems as contrast agents for magnetic resonance imaging.

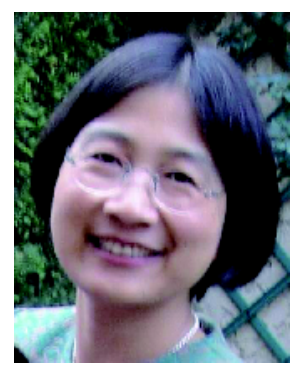

Ling Peng is currently a research director at the French National Scientific Research Center (CNRS), and a principal investigator at the Centre Interdisciplinaire de Nanoscience de Marseille in France. She is working actively at developing dendrimer nanosystems for biomedical applications, and has successfully established bio-inspired and self-assembling dendrimers for drug delivery. She is also interested in elaborating chemical probes to study biological events, and being active at the interface of chemistry and biology.

\section{树型分子的磁共振造影剂在脑肿瘤成像中的应用}

丁玲 ${ }^{1,2}$, 吕振斌 ${ }^{1}$, Dinesh Dhumal ${ }^{1}$, 高佳麟, ${ }^{3,4}$, Monique Bernard ${ }^{2}$, 彭玲 ${ }^{1 *}$

摘要 脑癌由于其特殊的生理位置和血脑屏障, 是一种难以治疗的致命肿瘤. 手术切除是目前脑癌的主要治疗手段, 然而成功的手术需要 精确的肿瘤定位和肿瘤成像以确保完全切除癌细胞并避免伤害正常脑组织. 磁共振成像是诊断脑癌最常见的成像方法, 但通常需要造影 剂来增强成像对比度和精准度. 树型分子因其独特的精准三维结构, 可调控的纳米尺寸和众多的表面官能团, 可作为构建磁共振成像造影 剂的优选材料. 藉由调整树型分子的尺寸、使用不同的分支结构和表面官能团修饰（包括磁共振成像功能团和脑肿瘤靶向基团）可以改 善造影剂的弛豫性, 克服血脑屏障并增强肿瘤靶向能力, 从而改善成像质量以获得精准的脑瘤成像, 并提高造影剂的安全性. 本文简要总 结了基于树型分子的磁共振成像造影剂在脑癌成像应用中的挑战、最新进展、机遇和未来发展趋势. 